\title{
Codon bias confers stability to human mRNAs
}

\section{AUTHOR(S):}

Hia, Fabian; Yang, Sheng Fan; Shichino, Yuichi; Yoshinaga, Masanori; Murakawa, Yasuhiro; Vandenbon, Alexis; Fukao, Akira; ... Adachi, Shungo; Iwasaki, Shintaro; Takeuchi, Osamu

\section{CITATION:}

Hia, Fabian ...[et al]. Codon bias confers stability to human mRNAs. EMBO reports 2019, 20(11): e48220.

\section{ISSUE DATE:}

2019-11-05

URL:

http://hdl.handle.net/2433/244816

\section{RIGHT:}

This is the author's version of the paper, which has been published in final form at

https://doi.org/10.15252/embr.201948220; The full-text file will be made open to the public on 3 March 2020 in

accordance with publisher's 'Terms and Conditions for Self-Archiving'; この論文は出版社版でありません。引用の際に

は出版社版をご確認ざ利用ください。; This is not the published version. Please cite only the published version, 


\section{Codon Bias Confers Stability to Human mRNAs}

2 Fabian $\mathrm{Hia}^{1}$, Sheng Fan Yang ${ }^{1}$, Yuichi Shichino², Masanori Yoshinaga ${ }^{1}$, Yasuhiro Murakawa ${ }^{3}$,

3 Alexis Vandenbon ${ }^{4}$, Akira Fukao ${ }^{5}$, Toshinobu Fujiwara ${ }^{5}$, Markus Landthaler ${ }^{6}$, Tohru Natsume ${ }^{7}$,

4 Shungo Adachi ${ }^{7}$, Shintaro Iwasaki ${ }^{2,8}$, and Osamu Takeuchi ${ }^{1, *}$

51 Department of Medical Chemistry, Graduate School of Medicine, Kyoto University, Kyoto 606-8501,

6 Japan

$7 \quad 2$ RNA Systems Biochemistry Laboratory, RIKEN Cluster for Pioneering Research, Wako, Saitama

8 351-0198, Japan

$9{ }^{3}$ Division of Genomic Technologies, RIKEN Center for Life Science Technologies, Yokohama,

10 Kanagawa 230-0045, Japan; RIKEN Preventive Medicine and Diagnosis Innovation Program,

11 Yokohama, Kanagawa 230-0045, Japan.

$12{ }^{4}$ Laboratory of Infection and Prevention, Institute for Frontier Life and Medical Sciences, Kyoto

13 University, Kyoto, 606-8507, Japan.

$14{ }^{5}$ Laboratory of Biochemistry, Department of Pharmacy, Kindai University, Higashiosaka City, Osaka, 15 577-8502 Japan

${ }^{6}$ RNA Biology and Posttranscriptional Regulation, Max Delbrück Center for Molecular Medicine Berlin, Berlin Institute for Molecular Systems Biology, 13125 Berlin, Germany; IRI Life Sciences, Institut für Biologie, Humboldt-Universität zu Berlin, 10115 Berlin, Germany.

${ }^{7}$ Molecular Profiling Research Center for Drug Discovery (molprof), National Institute of Advanced Industrial Science and Technology (AIST), Tokyo 135-0064, Japan

${ }^{8}$ Department of Computational Biology and Medical Sciences, Graduate School of Frontier Sciences, The University of Tokyo, Kashiwa, Chiba 277-8561, Japan

* To whom correspondence should be addressed. Tel: +81-75-753-9500; Fax: +81-75-753-9502;

Email: otake@mfour.med.kyoto-u.ac.jp

\section{Abstract}

Codon bias has been implicated as one of the major factors contributing to mRNA stability in several model organisms. However, the molecular mechanisms of codon-bias on mRNA stability remain unclear in humans. Here we show that human cells possess a mechanism to modulate RNA stability through a unique codon bias. Bioinformatics analysis showed that codons could be clustered into two distinct groups - codons with $\mathrm{G}$ or $\mathrm{C}$ at the third base position (GC3) and codons with either $\mathrm{A}$ or $\mathrm{T}$ at the third base position (AT3); the former stabilizing while the latter destabilizing mRNA. Quantification of codon bias showed that increased GC3 content entails proportionately higher GC content. Through bioinformatics, ribosome profiling and in vitro analysis, we show that decoupling the effects of codon bias reveals two modes of mRNA regulation, one GC3- and one GC-content dependent. Employing an immunoprecipitation-based strategy, we identify ILF2 and ILF3 as RNA binding proteins that differentially regulate global mRNA abundances based on codon bias. Our results demonstrate that codon bias is a two-pronged system that governs mRNA abundance. 


\section{Introduction}

Messenger RNA (mRNA) regulation represents an essential part of regulating a myriad of physiological processes in cells, being indicated in the maintenance of cellular homeostasis to immune responses [1-3]. In addition to transcription regulation, post-transcriptional regulation of mRNA stability is vital to the fine-tuning of mRNA abundance. To date, several mRNA-intrinsic properties, often in $5^{\prime}$ or $3^{\prime}$ untranslated regions (UTR), have been shown to affect mRNA stability $[4,5]$. Due to the recent advances in technology, the contribution of mRNA stability to gene expression has been suggested [6]. However, the regulation of mRNA stability, which is possibly governed by mRNA intrinsic features, has not been fully elucidated.

One of the most crucial mRNA-intrinsic features is codon bias. To scrutinize this bias in usage of redundant codons, several metrics to measure how efficiently codons are decoded by ribosomes (codon optimality) have been proposed. In a classical metric called the codon Adaptation Index (cAl), gene optimality is calculated by comparison between codon usage bias of a target gene and reference genes which are highly expressed $[7,8]$. Another index termed the tRNA Adaption Index (tAl) gauges how efficiently tRNA is utilized by the translating ribosome $[9,10]$. More recently, the normalized translation efficiency (nTE), which takes into consideration not only the availability of tRNA but also demand, was also proposed [11]. In addition to these, there are estimators of codon ribosome translation speed [12] as well as calculators of species-specific tAI [13].

Recently, Presnyak and colleagues showed that mRNA half-lives are correlated with optimal codon content based on a metric, the Codon Stabilization Coefficient (CSC) which was calculated from the correlations between the codon frequencies in mRNAs and stabilities of mRNAs. Additionally, they showed that the substitutions of codons with their synonymous optimal and non-optimal counterparts resulted in significant increases and decreases in mRNA stability in yeast [14]. This effect was brought by an RNA binding protein (RBP) Dhh1p (mammalian ortholog DDX6), which senses ribosome elongation speed [14-16]. In yeast, these differences in ribosome elongation speed in turn are influenced by tRNA availability and demand $[11,17,18]$. Taken together, codons can be designated into optimal and non-optimal categories; the former hypothesized to be decoded efficiently and accurately $[19,20]$ while the latter slow ribosome elongation resulting in decreased mRNA stability [14-16]. It is also important to make the distinction that common and rare codons do not necessarily imply optimal and non-optimal codons.

At present, codon optimality-mediated decay has been extensively studied and established particularly in Saccharomyces cerevisiae as well as other model organisms such as Schizosaccharomyces pombe, Drosophila melanogaster, Danio rerio, Escherichia coli, Trypanosoma brucei and Neurospora crassa [21-27]. At present, the molecular mechanisms of this system of codon optimality in humans are under intense scrutiny $[28,29]$.

In this study, we show that codon bias-mediated decay exists in humans. Principal component analysis (PCA) showed that codons could be clustered into two distinct groups; codons with $\mathrm{A}$ or $\mathrm{T}$ at 
the third base position (AT3) and codons with either $\mathrm{G}$ or $\mathrm{C}$ at the third base position (GC3). This clustering was associated with mRNA half-lives enabling us to determine GC3 and AT3 codons as stabilizing and non-stabilizing codons respectively. In this regard, the increased usage of GC3 codons entails an inevitable increase GC-content. We then developed an algorithm to quantify the codon bias of GC3 codons. With ribosome profiling, we show that codon bias-derived occupancy scores agreed with ribosome occupancy. Additionally, bioinformatics analysis revealed that frameshifts abrogate this GC3-AT3 delineation. We then verified our results in vitro using optimized and de-optimized reporter constructs. Here we propose that GC3 codons and AT3 codons are optimized and de-optimized codons respectively. Importantly, frameshifted optimized transcripts retain a certain level of stability suggesting that overall the overall GC content of transcripts is an additional determinant of stability. Finally, employing a ribonucleoprotein immunoprecipitation strategy, we identified RNA binding proteins which were bound to transcripts with low or high GC3-content. We propose that interleukin enhancer-binding factor 2 (ILF2) mediates mRNA stability of transcripts via codon bias.

\section{Results}

\section{Codons in Homo sapiens can be categorized into GC3 and AT3 codons}

To examine whether a system of codon bias exists in human, we first compared codon frequencies in Homo sapiens and other model organisms. Hierarchical clustering analysis of codon frequency data obtained from Ensembl database [30] showed a difference between lower eukaryotes such as Saccharomyces cerevisiae and Caenorhabditis elegans, and higher eukaryotes such as Homo sapiens and Mus musculus (Fig 1A). To investigate codon bias in humans, we downloaded human coding sequence (CDS) data from the Ensembl Biomart database and calculated the codon counts for each coding sequence. For each CDS, we calculated the codon frequencies by expressed the codon counts as a fraction of the total number of codons in the CDS. We then performed a principal component analysis (PCA) on the CDS codon frequencies. The first principal component (PC1) of the PCA which accounted for $22.85 \%$ of the total variance, divided codons into two clusters: codons with either $\mathrm{G}$ or $\mathrm{C}$ at the third base position (GC3) and codons with either A or T at the third base position (AT3) (Fig 1B). Interestingly, the division within the second principal component (PC2) appeared to be split along the number of $G / C$ or $A / T$ bases in codons. We repeated our analysis on the CDS sequences from S. cerevisiae and found no such clustering (Fig EV1A). However, we discovered that the factor loading scores of the codons along the first principal component of our analysis in yeast corresponded to the CSC metric [14], albeit differences in the order (Fig EV1B). The abovementioned results therefore raised the possibility that the PCA method might have identified optimal and non-optimal codons; GC3 and AT3 codons in humans may have a valid effect on mRNA stability. To investigate the agreement between the PCA method and CSC in humans, we calculated the CSC scores in humans using published datasets of global mRNA decay rates in physiologically growing HEK293 cells (GSE69153) [Data ref: 31,32] and compared them to the PC1 factor loading scores of the codons (Fig EV1C). We observed a correlation of $\mathrm{R}^{2}=0.58$ between the two outputs indicating a moderately strong agreement despite the methodologies being different. 
114 We then tested the link between mRNA stability and GC3-AT3 codons using the above-mentioned mRNA stability data (GSE69153) [Data ref: 31,32]. Briefly, we divided the transcripts equally into quartiles based on their half-lives and averaged the codon frequencies within the quartiles. Strikingly, genes with short half-lives were associated with AT3 codons while genes with longer half-lives were associated with GC3 codons (Fig 1C), suggesting a connection between third base of codons and the stability of mRNAs.

Broadly, the codon bias in mRNA can predict the stability of the mRNA. Classification by GC3-content might potentially implicate GC-content as a factor which might affect the stability of mRNA. By summing the GC3 frequencies and GC bases of CDS sequences, we could determine the GC3- and GC- content of a gene (Dataset EV1). We then visualized the genome-wide GC3 and GC landscape by plotting the corresponding values via a histogram (Figure 1D). GC3-content was represented as a bimodal distribution with a range of values from the minimum of $24.1 \%$ to the maximum of $100 \%$ while GC-content appeared similarly as a bimodal distribution with a range of values from a minimum of $27.6 \%$ to the maximum of $79.7 \%$. A Pearson correlation analysis $\left(R^{2}=0.869\right)$ between gene GCcontent and GC3-content (Fig EV1D) reflected an enrichment of GC-content with increased GC3content. Indeed, higher GC3-content was generally associated with better stability (Fig 1E top and Fig EV1E). To further verify the impact of GC3-content on mRNA stability, we plot the GC3-content data in Fig 1E (top) in the form of cumulative distribution functions and found these distributions to be significantly different from the genome average (Fig EV1F). As with our analysis with GC3-content, we grouped the half-life data by GC-content (Fig 1E, bottom) and observed a similar increase in halflives even with the GC-content grouping. Interestingly, we also noted a decrease in half-life beyond a GC-content of $60 \%$; this decrease also coinciding with the decrease in half-lives in the GC3-content grouping (Fig EV1D). While we are currently unable to explain the associated decrease in both plots at extreme GC3- and GC- content, it would be interesting to investigate this particular drop-off in stability in the future.

Additionally, we noted that the codon bias per se was different between yeast and humans (Fig 1B and Fig EV1A) [14]. We also observed this difference in Xenopus, zebrafish as well as Drosophila, when compared to humans [24,33]. We repeated our analysis, this time grouping the half-life dataset by their respective $\mathrm{CAI}$ (Fig EV1G). With the cAl dataset, we were able to observe increased half-life with an associated increased in cAl albeit only from the range of $0.75-0.95$. In contrast, the PCAderived GC3-content method was better able to recapitulate this increase in half-life compared to the cAl metric. Taken together, our analysis allowed us to designate GC3 and AT3 codons as stabilizing and destabilizing codons respectively. Additionally, high GC3 content in transcripts inevitably results in high GC-content, which is a feature of stable mRNAs.

We then asked about the biological relevance associated with codon bias. Taking the $5 \%$ of lowest and highest ranked genes into account, we observed that genes with high GC3-content were enriched in developmental processes while genes with low GC3-content were enriched in cellular division processes (Fig EV1H and I), suggesting the importance of codon bias-mediated mRNA decay across dynamic cellular processes in humans. 
154

155

156

157

158

159

160

161

162

163

164

165

166

167

168

169

170

171

172

173

174

175

176

177

178

179

180

181

182

183

184

185

186

187

188

189

190

191

Given that GC3-AT3 codons were associated with high and low stability respectively, we wondered if these two groups were synonymous with optimal and non-optimal codons. It has been proposed that slower ribosome elongation rate modulated by low codon optimality affects the stability of mRNAs in yeast [14]. This led us to examine whether decelerated ribosomes could be observed especially in regions where optimality was low. From the PCA, PC1 factor loadings of the codons were indicative of how much a particular codon contributed to the AT3-GC3 grouping i.e. instability-stability (Fig EV2A). Therefore as a measure of estimating ribosome occupancy, the factor loading scores of the codons from the first principal component were utilized to derive codon bias-derived occupancy scores (refer to materials and methods for details on the calculation of scores). Because we speculated that a single codon would be insufficient in eliciting any noticeable effects on the speed of the ribosome, we divided each CDS into 25 bins from start codon to stop codon and summed up the codon bias-derived occupancy scores. We then compared these scores with corresponding ribosome occupancies derived from ribosome profiling [34]. Ribosome occupancy obtained from HEK293 cells growing under physiological conditions generally coincided with codon bias-derived occupancy (Fig 2A). These measurements were highly reproducible between replicates of ribosome profiling experiments across the transcriptome $\left(R^{2}=0.750,16,423\right.$ transcripts) (Fig EV2B). We observed a significantly better prediction of ribosome occupancy by codon bias-derived occupancy scores than that derived from scrambled codon bias-derived occupancy scores (Fig 2B). Unfortunately, at the individual codon level, we only observed a weak but positive correlation $\left(R^{2}=0.13\right)$ between ribosome occupancy and codon-bias derived scores (Fig EV2C). We believe that this difference in both calculations can be attributed to the binning of the ribosome occupancy data which ensures that any reasonable slowing of ribosomes in regions of low optimality could be accurately manifested. Indeed, representative transcripts showed a good correlation between our binned codon bias-derived occupancy scores and ribosome occupancy as exemplified by EIF2B2, DYNC1LI2, and IDH3G transcripts (Fig EV2D).

Although translation elongation and initiation are distinct steps, previous literature has suggested that optimal codons are also enriched in mRNAs with high translation [35]. Ribosome footprint reads normalized by mRNA abundances from RNA-Seq enables the calculation of translation efficiency which in turn is also generally regarded as the translation initiation rate [36]. Therefore, to establish the link between translation status and codon bias, we calculated the translation efficiency (TE) ribosome footprints normalized by mRNA abundance. Indeed, our results showed that mRNAs with high GC3-content generally possessed high TE (Fig 2C). This phenomena also coincides with known research in zebrafish and yeast in that optimal genes generally have high TE [33,37]. To exclude the effect of mRNA abundances on TE, we grouped mRNA of similar abundances into separate groups and repeated our analysis (Fig EV2E). Within these groups, we still observed a general increase in TE within each of the groups, albeit a decrease in TE at a GC-content of $70-80 \%$ across all ranges of mRNA abundances (similar to Fig $\mathbf{2 C}$ ).

To verify if GC3 and AT3 codons were indeed associated with stability and instability respectively, we performed PCA on +1 and -1 frameshifted CDS sequences genome-wide and show that the GC3-AT3 
demarcation was abolished (Fig 2D and E). Interestingly, we found that GC-rich (two or three G/C bases) and AT-rich (two or three A/T bases) codons contributed strongly to PC1 of the frameshifted data showing that GC/AT content is a natural consequence GC3-AT3 usage (Fig 1D and Fig EV1D).

Thus far, we show that GC3 and AT3 codons are associated with mRNA stability, ribosome translation speed and efficiency therefore suggesting that the former and latter can be designated into optimal and non-optimal codons respectively.

\section{Codon bias affects mRNA stability}

We then experimentally validated our bioinformatics observations of GC3 and AT3 codons in human cells. We developed a scheme based on the PC1 factor loadings in which we previously utilized in our ribosome profiling analysis (Fig EV2A). Based on this scheme, codons could be optimized and deoptimized with regard to GC3 content within their codon boxes i.e. synonymous substitutions (Fig EV3A). Single box codons such as TGG (Trp) and ATG (Met) would remain unchanged. We synthesized two independent genes (REL and IL6) with differential GC3-content (Fig EV3B, Dataset EV2) and examined the stability of these reporter RNA in HEK293 cells utilizing the Tet-off system (Fig 3A). As expected, the optimized transcripts of REL and IL6 were more stable than their wild-type counterparts. Additionally, the decay rate of the de-optimized IL6 reporter was faster, confirming that low GC3-content transcripts were unstable.

In addition to the RNA stability, higher GC3-content was also associated with higher translation efficiency (Fig $\mathbf{2 C}$ ), thereby increasing protein production. Indeed, the protein abundance of the optimized REL reporter was higher than REL-WT even after normalization of protein abundance by steady state mRNA levels (Fig 3B, Fig EV3C). Using enzyme-linked immunosorbent assay (ELISA), we observed that expression of IL6-OPT resulted in a 1.5-fold and 2-fold significantly higher level of IL6 compared to its WT and IL6-DE, respectively (Fig 3C). In a similar fashion, normalization of IL6 protein abundance by mRNA levels revealed that translation efficiency of the optimized IL 6 reporter was higher than its WT and de-optimized reporter counterparts (Fig EV3D). We tested our REL reporters in HeLa cells and show that the high protein abundance of REL-OPT could also be observed (Fig EV3E). Similarly, actinomycin-based stability measurements of the REL reporters in HeLa cells revealed a similar increase in mRNA stability in the REL-OPT transcript (Fig EV3F). Moreover, polysome fractionation and subsequent $\mathrm{PPCR}$ analysis revealed that within the polysome fractions, REL-OPT transcript amounts were proportionately higher than REL-WT transcripts, suggesting that REL-OPT was translated more efficiently than REL-WT (Fig 3D). Thus far, our results validate the bioinformatics analyses and show that GC3 and AT3 codons can be designated as optimal and non-optimal codons.

\section{GC-content as an additional determinant of stability}

We then hypothesized that if the effect on mRNA stability was entirely the result of translational elongation, blocking translational elongation would restore stability to transcripts possessing low optimality to levels similar to that of their high optimality counterparts. We therefore treated cells 
expressing the REL reporters with a translation inhibitor, cycloheximide $(\mathrm{CHX})$, and assayed the mRNA decay rates via the Tet-off system (Fig 4A). Treatment with $\mathrm{CHX}$ improved the stability of both REL-OPT and REL-WT transcripts compared to the control group. Interestingly, the stability of CHXtreated REL-WT transcripts was still significantly lower than that of CHX-treated REL-OPT transcripts. We repeated our experiments using the IL6 reporters and found that in a similar fashion, CHX-treated IL6-DE transcripts were stabilized, albeit, not to the same extent as CHX-treated IL6-OPT (Fig 4B). Following this, we repeated our experiments using a different translation inhibitor, anisomycin (ANI) and obtained similar results (Fig $\mathbf{4 C}$ and $\mathbf{D}$ ), suggesting that a translation-independent mRNA degradation pathway could also be present. It should be noted that an important caveat to the use of global translation inhibitors, $\mathrm{CHX}$ in particular, is that they have been reported to potentially distort mRNA level measurements as well as translation efficiency [38-40].

We then synthesized $a+1$ frameshifted version of the REL-OPT transcript, removing any potential stop codons which would have resulted in premature termination of transcription and measured its stability via the Tet-off system (Fig 4E). This frameshifted version, while retaining a high GC-content (similar to REL-OPT), possessed a lower GC3-content, than its in-frame counterpart (Fig EV3B). Surprisingly, the frameshifted version, was still more stable than the WT form, yet less stable compared to its in-frame optimized counterpart, suggesting that high GC / low AU-content was able to retain a significant amount of transcript stability. To verify our findings, we similarly synthesized $a+1$ frameshifted version of the IL6-OPT transcript which had a high GC-content (similar to IL6-OPT) but a GC3-content of 39.15\%; the GC3-content falling between its WT and DE counterparts (Fig EV3B). This frameshifted version of IL6 was relatively more stable compared to the DE transcript (Fig $\mathbf{4 F}$ ). Taken together, our results reinforce the notion that in addition to GC3-content, GC-content could be an additional determinant of stability. Taken together, our results show that codon bias encompasses two modes of mRNA regulation, GC3- and GC-content dependent.

\section{RNA binding proteins differentially bind to transcripts of varying degrees of codon bias}

254

Having shown that high optimality content inevitably accords high GC-content which in turn promotes mRNA stability, we wondered if there were RNA binding proteins (RBPs) which scrutinize, discriminate or even affect an mRNA's fate. To identify RBPs which were either bound to transcripts bearing high or low optimality, we performed a ribonucleoprotein immunoprecipitation-based

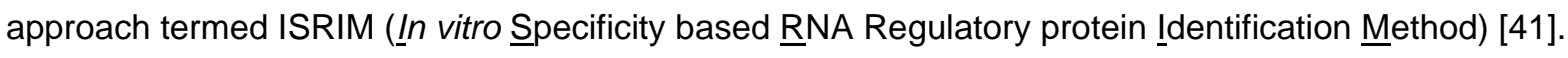
Lysates of HEK293 cells were mixed with FLAG peptide-conjugated REL and IL6 transcripts of high and low optimality and their interacting proteins were determined using mass spectrometry. We then calculated the fold changes based on the abundance of RBPs bound to REL-WT with respect to RELOPT (Fig 5A).

As IL6 transcripts possessed three levels of GC3-content (OPT, WT, DE), we defined high GC3content binding RBPs based on the RBP enrichment of IL6-DE to IL6-WT (Fig 5B) as well as IL6-WT compared to IL6-OPT (Fig 5C). Similarly, we defined low GC3-content binding RBPs based on the RBP enrichment of IL6-DE compared to IL6-WT (Fig 5B) as well as IL6-WT to IL6-OPT (Fig 5C). By 
selecting common RBPs belonging to each group, we defined a set of RBPs which bound differentially to high GC3 and low GC3 IL6 transcripts respectively (Fig EV4A) We then selected RBP candidates which were specifically enriched with either low or high GC3 transcripts common to both REL and IL6 ISRIM experiments (Fig EV4B, Dataset EV3). In all, we show that RBPs can differentiate between transcripts of high GC3- and low GC3- content.

\section{ILF2 regulates the stability of low GC3 / high AT3 transcripts}

We investigated the role of RBPs in modulating the stability of transcripts with different codon bias. Of interest were ILF2 and ILF3, RPBs identified from the list of RBPs interacting exclusively with low optimality transcripts. ILF2 and ILF3, also known as NF45 and NF90/NF110, respectively, are well known to function dominantly as heterodimers which bind double stranded RNA. ILF3 has been extensively studied, having shown to bind to AU-rich sequences in 3' UTR of target RNA to repress its translation [42]. We hypothesize that the binding of ILF2 and ILF3 as a heterodimer to their targets occur as low optimality transcripts are inadvertently AU-rich. Here we focused on the effects of these RBPs on low optimality transcripts. Firstly, using published RIP-seq data of ILF2 in two multiple myeloma cell lines, H929 and JJN3, we observed that ILF2, interacts with low optimality transcripts (Fig EV5A) [Data ref: 43,44]. Additionally, we analysed RNA-Seq data obtained from the ENCODE project of K562 cells treated by CRISPR interference targeting ILF2 [Data ref: 45]. Strikingly, we observed that transcripts that possessed low optimality scores were upregulated whereas transcripts that possessed high optimality scores were downregulated (Fig 6A, Fig EV5B). The abundance changes of representative mRNAs by ILF2 knockdown were antiparallel to their GC3-content (Fig EV5C).

However, differences in mRNA levels do not necessarily imply a difference in mRNA stability. To confirm if mRNA stability was indeed affected, we examined the stability of FLAG-tagged versions of REL-OPT and REL-WT in the Tet-off system after ILF2 and ILF3 knockdown via siRNA (Fig 6B-C). Interestingly, we observed that the optimized reporter was more unstable under the knockdown of both ILF2 and ILF3 whereas the WT reporter was more stable with the knockdown of ILF2 and a combination of both ILF2 and ILF3 knockdown. In agreement with this, we found a significant increase in protein levels of REL-WT when cells were treated with ILF2- and ILF3-targeting siRNA (Fig 6D and Fig EV5D). However, despite seeing a decrease in stability of the GC3-optimized reporter under both ILF2 and ILF3 knockdown, we were unable to observe this change at the protein level. Focusing our attention on ILF2, we expressed FLAG-tagged versions of REL-OPT and REL-WT, along with the two isoforms of ILF2 and detected the reporter protein levels via western blot. A significant decrease in band intensity was observed for the REL-WT bands when both isoforms of ILF2 were expressed, whereas the amount of REL-OPT was not changed (Fig 6E and Fig EV5E). Taken together, our results suggest that ILF2 and ILF3 affect mRNA transcripts with low GC3-content (and inadvertently low GC-content) to induce their decay.

Next, we sought to identify possible motifs which are enriched in ILF2/3 targets. Based on the RIPseq data in JJN3 and H929 [Data ref: 43,44], we identified common transcripts which were more than 
5 -fold differentially upregulated and subjected their cDNA sequences to de novo motif identification via the MEME (Multiple EM for Motif Elicitation) software [46]. Our analysis identified AU-rich motifs of about 6-7nt long (Fig EV5F) as well as their distributions mainly in the CDS and 3'UTR along target transcripts. It should be noted that that these motifs are enriched in mRNA targets, and may not necessarily imply bona fide binding motifs of ILF2/3. Therefore, we performed an additional motif search on a recently identified and experimentally validated ILF3 motif from RNA Bind-n-seq experiments by Dotu and colleagues [47] and found a similar distribution of motifs in the CDS and 3'UTR of targets (Fig EV5F).

\section{Discussion}

This study provides a framework describing codon bias-mediated RNA decay in humans. We first show that GC3 codons are associated with stability and AT3 codons with instability. We quantified codon bias by calculating the GC3 content within the CDS of genes and showed that GC3-content is strongly correlated with RNA stability and amount of protein expressed. In general, the use of optimal GC3 codons correlated with higher GC-content at a genome-wide level. We then show a modest agreement between codon bias-derived scores and ribosome occupancy as determined by ribosome profiling. Using GC3-optimized and de-optimized reporters we validate our bioinformatics observations in vitro. Screening of RNA binding proteins and further in vitro analysis suggests a role of ILF2, possibly in complex with ILF3, in the codon-mediated regulation of mRNA. Taken together, we conclude that gene expression can be shaped by codon bias and inevitably by GC/AU-content through the modulation of mRNA stability in human cells.

\section{Investigating the System of Codon Bias in Humans}

Since translation elongation is affected by tRNA availability, the tRNA adaptation index (tAl), which is based on genomic tRNA copy number, has been used as a surrogate for codon optimality. However, in contrast to yeast, tRNA copy number in the genome is not always correlated with tRNA abundance in higher eukaryotes [48]. Hence, this metric is less suitable for quantifying codon optimality in humans. Independent of tRNA-based metrics, we addressed these challenges by utilizing an unsupervised learning algorithm, PCA, to identify features in that were mRNA-intrinsic. In the PCA of both yeast and humans, we demonstrated that the first principal component mirrored optimal/nonoptimal assignments. We also show that the codon bias is different between these two organisms (Fig 1B, Fig EV1A). In humans, the classification of codons into AT3 and GC3 groups was striking, but the percentage by which it accounts for its variation however was modest.

From the PCA, the first and second principal components only explain a quarter of total variance in codon frequencies (Fig 1B), implying that other factors that explain bias of codon frequency possibly remains in human cells. The limitation of this method is reflected in the use of codon frequencies as our input data for the PCA. This approach might have neglected other factors of stability or instability which might be codon-independent or which might be inherent at the nucleotide level. Assuming that evolution drives the selection of codons, synonymous codon usage in different organisms must be 
342 fine-tuned over time to achieve precise expression levels of mRNA and eventually proteins in

343 essential physiological process. Indeed, similar to our findings, a study by Bazzini et al. showed that a system of codon optimality is conserved among vertebrates, Xenopus and Zebrafish [33]. In addition, they demonstrated that in Zebrafish embryos, low codon optimality was associated with shorter poly $(A)$ tail length in addition to lower levels of translation. Our data together with recently published work by Wu and colleagues [28] indicates that a system of codon optimality exists in humans.

Our investigations show that high GC3/AT3-content or GC/AT-content in mRNA is selected for to modulate transcript stability in essential physiological processes, but is subject to constraints by amino sequence. Indeed, we show that transcripts with high and low GC3-content were linked to particular physiological and cellular processes (Fig EV1H and I). In a particular study, Gingold and colleagues argue that tRNA abundances vary in proliferating and differentiating cell types [49]. Interestingly, they showed that codons preferred by cell cycling genes were AT3 codons while pattern-specification preferred codons tended to be GC3 codons-in agreement with our GO analyses. In Drosophila, the correlation between codon optimality and mRNA stability has been demonstrated to be attenuated in neural development, possibly allowing the effect of trans-acting factors to dominate development [24].

Our results show that the codon bias we have identified affects ribosome occupancy to a significant but limited extent (Fig 2B). At the level of individual codon occupancies, we only observed a weak but positive correlation $\left(R^{2}=0.13\right)$ between ribosome occupancy and codon-optimality derived scores (Fig EV2C). These results however are not surprising given that studies based on ribosome profiling data found no correlations between ribosome occupancy and rare codons [50,51]. In view of this we binned the CDS into 25 evenly spaced groups to ensure that any reasonable slowing of ribosomes in regions of low optimality could be accurately represented by the GC3-AT3 bias. However, we acknowledge that our matric is only able to demonstrate a prediction to a limited extent. There are many factors can affect ribosome profiling results such as growth conditions, coverage, cloning and sequencing biases, methods of bioinformatic analysis, as well as experimental noise $[18,52,53]$.

Taking into account our in vitro experiment results together with the ribosomal profiling results, we suggest that GC3 and AT3 codons are synonymous with optimal and non-optimal codons. Additionally, our study along with others' suggests that slower elongation of ribosome is a key feature of mRNA stability. However, it should be noted that in our analysis methodology, the assumption that stability is solely a function of ribosome speed might only hold true to a limited extent. There is evidence to show that mRNA intrinsic features which have the propensity to regulate ribosome velocity are essential in maintaining the function and correct expression of proteins, the failure of which may result in degradation of the mRNA and protein: Although codon optimality is a dominant factor in general, other factors may also be involved in decelerated ribosomes, such as secondary structures [54,55]. These obstacles for ribosome elongation are reversible and dynamically regulated by RNA helicases [56,57]. Importantly, these structures may serve to reduce ribosome speed when 
been shown in Neurospora that codon usage can regulate co-translational protein folding and subsequently, its function [59].

As such, while we have shown that the optimizations of transcripts leads to increases in protein production, further studies are required to investigate protein folding dynamics and determine if the produced protein still retains its functionality. Furthermore, in a study of two model organisms, E. coli and S. cerevisiae by Tuller et. al., the rate of translation elongation was shown to be determined by the folding energy, codon bias and amino acid charge of at the beginning of the CDS [60]. It is likely that these factors may also affect the local speed of the ribosome further down the CDS, and by extension, the stability of the mRNA. Further studies will be required to elucidate the role of RNA secondary structures and helicases and their relevance to codon bias, protein folding and mRNA stability decay.

In attempts to quantify the effect of ribosomal density on mRNA stability, several studies have demonstrated that in general, increased ribosomal density results in increased mRNA stability of a transcript $[61,62]$. This phenomenon has been attributed to competition between the initiation complex and decay factors as well as ribosomes sterically excluding decay factors from accessing the mRNA $[63,64]$. To this effect, reduction in translation initiation has been shown to decrease ribosomal density and subsequently, mRNA stability [65]. On the other hand, inhibiting translation elongation causes an increase in ribosome density and consequently, mRNA stability [66]. Here we show that optimized transcripts are highly polysome bound as opposed to their WT counterparts suggesting increased rates of translation initiation (Fig 3D). This is corroborated by our ribosome profiling findings that high GC3-containing transcripts have higher TE (Fig $\mathbf{2 C}$ ), possibly protecting transcripts from decay factors.

In this regard, transcripts with high optimality have higher translation initiation rates, causing them to be highly polysome bound. Additionally, optimized codons allow for efficient decoding and thus, smoother ribosome traffic. On the other hand, transcripts with low optimality tend to be less polysome bound with frequent ribosome deceleration and/or stalling. Our ribosome profiling analyses in Fig 2B however, is tailored to comparing the relative ribosome densities (in bins) within an individual transcript, against the codon bias-optimality scores. While we show relative accumulation of ribosomes in low optimality regions locally within a transcript, this particular analysis can neither be extended to comparing total ribosome densities across the transcriptome nor compared to the polysome profiling results.

Interestingly, in a separate study in Neurospora, gene expression modulated by codon usage was shown to be due to the effects of transcription rather than translation [67]. In a follow-up study, the group also demonstrated $\mathrm{C} / \mathrm{G}$ bias is able to promote gene expression by suppressing premature transcription termination [68]. In addition, several other studies have demonstrated that in mammalian cells, GC-rich genes are transcribed with increased efficiency resulting in higher levels of transcripts independent of mRNA degradation [69,70]. Next, a study by Fu et al. which investigated the effects of codon usage bias on two proto-oncogenes with similar amino acid identity, but differing levels of optimality, KRAS and HRAS, showed that codon usage can affect both transcription and translation 
efficiency suggesting that the effect of codon bias is multi-level [71]. In this and another study, changing the rare codons of KRAS to common ones increased its enrichment in the polysome fractions [72]. Likewise, REL-OPT transcripts were enriched in the polysome fractions compared to REL-WT transcripts. Nevertheless, our investigations also show that steady state transcript copy number of the optimized reporter transcripts were significantly higher than that of the WT (and DE versions) (Fig EV3C,D). In addition to this however, we also show increased translation efficiency in mRNA that contain a higher proportion of optimized codons. In our study and several other vertebrates however, translation is the predominant effector of gene expression [33].

At the time of writing this manuscript, a study was published by Wu and colleagues which demonstrated that translation is indeed a determinant of mRNA stability in human cells [28]. While paper by Wu et al. had assigned optimal and non-optimal designations to codons via the calculation of the CSC derived from ORFeome and SLAM-seq experiments, we noted that some of the findings paralleled ours. Indeed, the codon designations of optimal and non-optimal codons also showed modest delineation of codons into GC3 and AT3 codons respectively. In another article published in the bioRxiv preprint server, Forrest and colleagues utilized a combination of endogenous and human ORFeome collection mRNAs in human cells to derive the CSC for human cells [29].Similar to the study by Wu and colleagues, the codon designations of optimal and non-optimal codons also showed a modest division of codons into GC3 and AT3 codons respectively. Similarly, we also show that the use of optimal and non-optimal codons can affect both mRNA stability as well as translation initiation to a large extent (Fig 1-3); albeit transcription to a limited extent. However, we have yet to identify an RBP that is involved in direct co-translational decay of mRNAs in humans as with that in yeast. Moreover, DDX6, the mammalian ortholog of $\mathrm{DHH}$, was recently demonstrated in humans to be involved in miRNA-driven translational repression, not mRNA destabilization as previously shown in yeast [73]. DDX6 aside, it would certainly be exciting for future experiments to uncover the nature of this elusive RBP.

c-Rel, a protein encoded by the $R E L$ gene and a canonical nuclear factor $\mathrm{KB}$ (NF-KB) subunit, is expressed abundantly in differentiated lymphoid cells and has been shown to be vital in thymic regulatory $T$ cell development in addition to controlling cancer via activated regulatory $T$ cells $[74,75]$. Given the inherent low optimality and associated instability of $R E L$ in its WT form (Fig 3A), we wonder if besides transcriptional control of $R E L$, could there be other post-transcriptional regulation systems at play. Further studies would be necessary to investigate if codon optimality or codon optimalityassociated RBPs modulate REL gene expression.

In our investigation, mRNA stability can be affected by GC3- and GC- content. It is important to note that the latter of which is also implicated in several processes such as miRNA binding, mRNA folding and splicing which in turn can affect mRNA stability. It is thus plausible that GC-content can also affect gene expression independent of RBP association. A study of transcriptome miRNA binding sites has shown that effective miRNA binding sites tend to dwell in G-poor and U-rich environments [76]. In addition, while our analyses are CDS-based, it has been shown that GC-content of both introns and exons are important in splicing via RNA structures [77-79]. Taken together, we propose 
457 that codon bias is able to exert its effects at multiple levels, consequently effecting gene and protein

458 expression.

\section{The stability of mRNA can be modulated by RBPs which bind AU-rich sequences}

460

461

462

463

464

465

466

467

468

469

470

471

472

473

474

475

476

477

478

479

480

481

482

483

484

485

486

487

488

489

490

491

492

493

494

495

Whereas AU-rich elements (AREs) in the 3' UTR have been traditionally targeted by RBPs, we found that coding regions are also targeted by ARE-recognizing RBPs. The identification of the heterodimeric complex consisting of ILF2 and ILF3 among others shows that a wide array of RBPs recognizes low optimality (AU-rich) sequences (Fig 5). However, the binding of ILF2/3 to target RNA presents as a challenge when trying to identify its target motif. Studies have shown that the RNAbinding portion of the ILF2/3 complex, ILF3, in particular is a promiscuous RBP, binding to RNA with no obvious sequence specificity [80]. It is interesting to note that several binding motifs, all of which are AU-rich have been proposed for ILF3. Analysis of ILF3 RNA Bind-n-Seq measurements identified a 9nt AU-rich motif that is bound to by ILF3 [47]. Kuwano and colleagues show that NF90, the shorter isoform of ILF3, specifically targets a 30nt AU-rich sequence in mRNA 3'UTRs and represses their translation, not stability [42]. This state of promiscuousness was compounded by a recent study by Wu and colleagues, in which where almost all genes where ILF3 occupancy was detected on the genome by ChIP-seq, was ILF3 occupancy on the corresponding transcript. Indeed, ILF3 is a multifunctional protein, affecting several biological processes. In addition to ours, other studies have shown that ILF3 can contribute to splicing [81], stabilization, nuclear export [82] and as mentioned, translation [42].

ILF2 on the other hand has been less scrutinized compared to its partner. From our experiments, we find that the longer isoform of ILF2 is predominantly and highly expressed while the shorter isoform is low in expression. Additionally, we observed that overexpression of the longer isoform appeared to upregulate the expression of the shorter isoform albeit to a small extent. From the literature it is known that ILF2 stabilizes ILF3 in the heterodimeric form [83]. We postulate that it is possible that the ILF2/3 heterodimer represses translation of mRNA with AU-rich sequences at a steady state in both CDS and 3'UTR. Knockdown of ILF2/3 relieves the repression on translation initiation allowing an increase in bound (translating) ribosomes which sterically exclude decay factors from accessing the mRNA, thereby increasing stability. Indeed, the knockdown of ILF2, which is critical in maintaining the stability of the heterodimeric complex, results in a stabilization of mRNA possibly due to increased ribosome traffic. At the protein level, while the knockdown of ILF2 results in an increased protein expression of target mRNA, the combined effect of both ILF3 and ILF2 knockdown results in a higher increase in target mRNA expression as compared to the ILF2-only knockdown. Unfortunately, in the case of the ILF2/3 siRNA experiments (Fig 6D), we were unable to achieve a complete knockdown of ILF2 due to the very high and constitutive production of ILF2. However, we still noted a small reduction in ILF3 protein levels hinting that ILF2 stabilizes ILF3 in the heterodimer form. In addition, taking into consideration reports that ILF2 and ILF3 can function independently of each other [84-86], it is also possible that ILF2 and ILF3 regulate the fate of mRNA differently; ILF2 being able to dimerize with other binding partners such as ZFR and SPNR. It is unknown however, how optimized transcripts are affected. Whereas our screens revealed that ILF2/3 bind exclusively to low optimality targets, we 
noted from our analysis of ILF2 knockdown data from the ENCODE database [Data ref: 45] as well as tests from our reporter constructs that high optimality transcripts are being regulated. Given this, we postulate that ILF2/3 might not interact directly with high optimality targets. Instead, ILF2/3 may be indirectly (de)antagonizing certain transcripts which may code for other regulators of high optimality genes. Further investigations will be required to assess how high optimality transcripts are antagonized.

Our screens also detected HNRNPD/AUF1, which destabilizes transcripts via recognition of AU-rich motifs [87], binding to low optimality mRNAs (Dataset EV3). These observations emphasize the importance of AU-content, which is strongly connected with low optimality, in RNA destabilization. However, it is possible that these factors induced the degradation of AU-rich transcripts different from the model proposed by Presnyak and Radhakrishnan $[14,15]$ as our RBP identification method was not fully reflective of the active translational status required for co-translational degradation of mRNA transcripts. Further studies would be necessary to discern if these or other factors act as sensors of codon optimality during translation.

In conclusion, in human cells, the redundancy of the genetic code allows the choice between alternative codons for the same amino acid which may exert dramatic effects on the process of translation and mRNA stability. In our experiments, we show that two modes of mRNA regulation exist - GC3 and GC-content dependent. This system potentially confers freedom for calibrating protein and mRNA abundances without altering protein sequence. Beginning from our exploratory analysis, we have developed an approach to quantify codon bias and demonstrate that beneath the redundancy of codons, exists a system which modulates mRNA and consequently, protein abundance.

\section{Materials and Methods}

\section{Cell Cultures, Growth, and Transfection Conditions}

HEK293T cells were maintained in Dulbecco's modified eagle medium (DMEM) (Nacalai Tesque), supplemented with 10\% (v/v) fetal bovine serum. HEK293 Tet-off cells were maintained in Minimum Essential Medium Eagle - Alpha Modification (a-MEM) (Nacalai Tesque), supplemented with $10 \%(\mathrm{v} / \mathrm{v})$ Tet-system approved fetal bovine serum (Takara Bio) and $100 \mu \mathrm{g} / \mathrm{ml}$ of G418 (Nacalai Tesque). For REL and IL6 overexpression experiments, plasmids were transfected using PEI MAX (Polysciences Inc). For co-transfection of ILF2 siRNA with REL plasmids, Lipofectamine 2000 was used as per manufacturer's protocol. ILF2 siRNA which targeted ILF2 at exons 8 and 9 were Silencer Select siRNA, S7399 (Ambion, Life Technologies). Actinomycin D-based stability assays in HeLa cells were performed by adding actinomycin $D$ to the transfected cells to a final concentration of $2 \mu \mathrm{g} / \mathrm{ml}$.

\section{Plasmid Construction}

Codon optimized-REL (REL-OPT), IL6 (IL6-OPT) and codon de-optimized IL6 (IL6-DE) sequences were synthesized as gBlocks Gene Fragments (Integrated DNA Technologies) (Dataset EV2). The REL-OPT (+1 Frameshift) sequence was constructed by adding a +1 frameshift just after the start codon. Resulting stop codons were removed to ensure no premature termination. These sequences 
and corresponding WT sequences were polymerase chain reaction (PCR) amplified (with the inclusion of a FLAG tag for REL sequences) and inserted into the pcDNA3.1(+) vector (Invitrogen) and PTRE-TIGHT vector (Takara Bio). The sequences were confirmed via restriction enzyme digest and sequencing.

\section{Tet-Off Assay}

HEK293 Tet-off cells (Clontech) were transfected with pTRE-TIGHT plasmids bearing the (de)optimized and WT sequences and incubated overnight at $37^{\circ} \mathrm{C}$. Transcriptional shut-off for the indicated plasmids was achieved by the addition of doxycycline (LKT Laboratories Inc.) to a final concentration of $1 \mu \mathrm{g} / \mathrm{ml}$. Cycloheximide-based stability assays in HEK293 Tet-off cells were performed by adding actinomycin $D$ to the transfected cells to a final concentration of $50 \mu \mathrm{g} / \mathrm{ml}$. Anisomycin-based stability assays in HEK293 Tet-off cells were performed by adding anisomycin to the transfected cells to a final concentration of $20 \mu \mathrm{g} / \mathrm{ml}$. Samples were harvested at the indicated timepoints after the addition of doxycycline (and cycloheximide/anisomycin).

\section{RNA Extraction, Reverse Transcription PCR, and Quantitative Real-time PCR}

Total RNA was isolated from cells using TRIzol reagent (Invitrogen) as per manufacturer's instructions. Reverse transcription was performed using the ReverTra Ace qPCR RT Master Mix with gDNA remover kit (Toyobo) as per manufacturer's instructions. cDNA was amplified with PowerUp SYBR Green Master Mix (Applied Biosystems) and quantitative real-time PCR (qPCR) was performed on the StepOne Real-Time PCR System (Applied Biosystems). To quantify transcript abundance of the REL reporters, pTRE-TIGHT plasmids bearing the (de)optimized and WT reporter sequences were used as standards. Human GAPDH abundance was used for normalization. The list of qPCR primers can be found in Dataset EV2.

\section{Sucrose Gradient Centrifugation (Polysome Profiling)}

HEK293T were transfected with equal concentrations of REL-OPT and REL-WT plasmids. Cells were lysed the next day in polysome buffer [20 mM 4-(2-hydroxyethyl)-1-piperazineethanesulfonic acid (HEPES-KOH) (pH 7.5], 100 mM KCl, 5 mM MgCl $2,0.25 \%$ (v/v) Nonidet P-40, $10 \mu \mathrm{g} / \mathrm{ml}$ cycloheximide, 100 units/ml RNase inhibitor, and protease inhibitor cocktail (Roche)]. Lysates were loaded on top of a linear $15 \%-60 \%$ sucrose gradient [ $15 \%-60 \%$ sucrose, $20 \mathrm{mM} \mathrm{HEPES-KOH} \mathrm{[pH}$ 7.5], $100 \mathrm{mM} \mathrm{KCl}, 5 \mathrm{mM} \mathrm{MgCl}, 10 \mu \mathrm{g} / \mathrm{ml}$ cycloheximide, 100 units/ml RNase inhibitor, and protease inhibitor cocktail (Roche)]. After ultracentrifugation at $38,000 \mathrm{rpm}$ for $2.5 \mathrm{~h}$ at $4^{\circ} \mathrm{C}$ in a HITACHI P40ST rotor, fractions were collected from the top of the gradient and subjected to UV-densitometric analysis. The absorbance profiles of the gradients were determined at $254 \mathrm{~nm}$. For disassociation of ribosome and polysome, EDTA was added to $\mathrm{Mg}^{2+}$-free polysome buffer and $15 \%-60 \%$ sucrose gradient at concentrations of $50 \mathrm{mM}$ and $20 \mathrm{mM}$, respectively. For RNA analysis, RNA from each fraction was extracted via the High Pure RNA Isolation Kit (Roche) and subject to reverse transcription and qPCR.

\section{Immunoblot Analysis}

Samples were lysed in RIPA buffer (20 mM Tris-HCl [pH 8], $150 \mathrm{mM} \mathrm{NaCl}, 10 \mathrm{mM}$ EDTA, 1\% Nonidet-P40, $0.1 \%$ SDS, $1 \%$ sodium deoxycholate, and cOmplete Mini EDTA-free Protease Inhibitor 
571 Cocktail [Roche]). Protein concentration was determined by the BCA Protein Assay (Thermo Fisher).

572 Whole cell lysates were resolved by SDS-PAGE and transferred onto PVDF membranes (Bio-Rad).

573 The following antibodies were used for immunoblot analysis: mouse monoclonal anti-FLAG (F3165,

574 Sigma), mouse monoclonal anti-ILF2 (sc-365283, Santa Cruz Biotechnology), mouse anti- $\beta$-actin (sc-

575 47778, Santa Cruz), and mouse IgG HRP linked F(ab')2 fragment (NA9310, GE Healthcare).

576 Luminescence was detected with a luminescent image analyser (Amersham Imager 600; GE

577 Healthcare).

\section{ELISA}

579 HEK293T cells were transfected with pcDNA3.1(+) plasmids bearing the (de)optimized and WT

580 sequences and incubated overnight at $37^{\circ} \mathrm{C}$. Cell supernatant was aspirated and the cell monolayer

581 washed with $1 \times$ PBS (pre-warmed at $37^{\circ} \mathrm{C}$ ). Pre-warmed DMEM was added to the monolayer and the

582 cells incubated for $2 \mathrm{hr}$ at $37^{\circ} \mathrm{C}$. Thereafter, the cell supernatant was harvested and centrifuged at 300

$583 \mathrm{x} \mathrm{g}$ to pellet residual cells. The resulting supernatant was decanted and the concentration of secreted

$584 \quad$ IL6 was measured by the human IL6 ELISA kit (Invitrogen) according to the manufacturer's

585 instructions.

ISRIM (In vitro Specificity based RNA Regulatory protein Identification Method)

587 Preparation of bait RNAs. T7-tagged CDNA template was PCR amplified and subjected to in vitro transcription using a MEGAscript T7 kit (Applied Biosystems). Amplified cRNA was purified with an RNeasy Mini Kit (Qiagen) and then subjected to FLAG conjugation as described (10) with some modifications. Briefly, $60 \mu \mathrm{l}$ of freshly prepared $0.1 \mathrm{M} \mathrm{NalO}_{4}$ was added to $60 \mu \mathrm{l}$ of $250 \mathrm{pmol}$ cRNA, and the mixture was incubated at $0^{\circ} \mathrm{C}$ for $10 \mathrm{~min}$. The $3^{\prime}$ dialdehyde RNA was precipitated with $1 \mathrm{ml}$ of $2 \% \mathrm{LiClO}_{4}$ in acetone followed by washing with $1 \mathrm{ml}$ acetone. The pellet was dissolved in $10 \mu \mathrm{l}$ of 0.1 $\mathrm{M}$ sodium acetate, $\mathrm{pH} 5.2$ and then mixed with $12 \mu \mathrm{l}$ of $30 \mathrm{mM}$ hydrazide-FLAG peptide. The reaction solution was mixed at room temperature for $30 \mathrm{~min}$. The resulting imine-moiety of the cRNA was reduced by adding $12 \mu \mathrm{l}$ of $1 \mathrm{M} \mathrm{NaCNBH}_{3}$, and then incubated at room temperature for $30 \mathrm{~min}$. The RNA was purified with an RNeasy Mini Kit (Qiagen).

Purification and analysis of RNA-binding proteins. Purification and analysis of RNA-binding protein (RBP) were carried out as described [41] with some modifications. Briefly, HEK293T cells were lysed with lysis buffer [10 mM HEPES (pH 7.5), $150 \mathrm{mM} \mathrm{NaCl}, 50 \mathrm{mM} \mathrm{NaF}, 1 \mathrm{mM} \mathrm{Na}_{3} \mathrm{VO}_{4}, 5$ $\mu \mathrm{g} / \mathrm{ml}$ leupeptin, $5 \mu \mathrm{g} \mathrm{ml}$ aprotinin, $3 \mu \mathrm{g} / \mathrm{ml}$ pepstatin A, $1 \mathrm{mM}$ phenylmethylsulfonyl fluoride (PMSF), and $1 \mathrm{mg} / \mathrm{ml}$ digitonin] and cleared by centrifugation. The cleared lysate was incubated with indicated amounts of FLAG-tagged bait RNA, antisense oligos and FLAG-M2-conjugated agarose for $1 \mathrm{hr}$. The agarose resin was then washed three times with wash buffer [10 mM HEPES (pH 7.5), $150 \mathrm{mM} \mathrm{NaCl}$, and $0.1 \%$ Triton X-100] and co-immunoprecipitated RNA and proteins were eluted with FLAG elution buffer [0.5 mg/ml FLAG peptide, $10 \mathrm{mM}$ HEPES (pH 7.5), $150 \mathrm{mM} \mathrm{NaCl}$, and 0.05\% Triton X-100]. The bait RNA associated proteins were digested with lysyl endopeptidase and trypsin. Digested peptide mixture was applied to a Mightysil-PR-18 (Kanto Chemical) frit-less column (45 $30.150 \mathrm{~mm}$ ID) and separated using a $0-40 \%$ gradient of acetonitrile containing $0.1 \%$ formic acid for $80 \mathrm{~min}$ at a 
$5600+;$ AB Sciex). MS and MS/MS spectra were obtained using the information-dependent mode. Up to 25 precursor ions above an intensity threshold of 50 counts/s were selected for MS/MS analyses from each survey scan. All MS/MS spectra were searched against protein sequences of RefSeq (NCBI) human protein database using the Protein Pilot software package (AB Sciex) and its decoy sequences then selected the peptides FDR was $<1 \%$. Ion intensity of peptide peaks ware obtained using Progenesis QI for proteomics software (version 3 Nonlinear Dynamics, UK) according to the manufacturer's instructions.

\section{Ribosome profiling and RNA-Seq}

618 Ribosome profiling was performed according to the method previously described with following 619 modifications [34]. RNA concentration of naïve HEK293T lysate was measured by Qubit RNA BR

620 Assay Kit (Thermo Fisher Scientific). The lysate containing $10 \mu \mathrm{g}$ RNA was treated with $20 \mathrm{U}$ of 621 RNase I (Lucigen) for $45 \mathrm{~min}$ at $25^{\circ} \mathrm{C}$. After ribosomes were recovered by ultracentrifugation, RNA 622 fragments corresponding to 26-34 nt were excised from footprint fragment purification gel. Library

623 length distribution was checked using a microchip electrophoresis system (MultiNA, MCE-202, 624 Shimadzu).

625 For RNA-seq, total RNA was extracted from the lysate using TRIzol LS reagent (Thermo Fisher 626 Scientific) and Direct-zol RNA Kit (Zymo research). Ribosomal RNA was depleted using the Ribo-Zero 627 Gold rRNA Removal Kit (Human/Mouse/Rat) (Illumina) and the RNA-seq library was prepared using 628 TruSeq Stranded mRNA Library Prep Kit (Illumina) according to the manufacturer's instructions. The libraries were sequenced on a HiSeq 4000 (Illumina) with a single-end 50 bp sequencing run. 630 Reads were aligned to human hg38 genome as described $[34,88]$. The offsets of A-site from the $5^{\prime}$ end of ribosome footprints were determined empirically as 15 for 25-30 nt, 16 for 31-32 nt, and 17 for $33 \mathrm{nt}$. For RNA-seq, offsets were set to 15 for all mRNA fragments. For calculation of the ribosome occupancies, mRNAs with lower than one footprint per codon were excluded. For calculation of the translation efficiencies (TEs), we counted the number of reads within each CDS, and ribosome profiling counts were normalized by RNA-seq counts using the DESeq package [89]. Reads corresponding to the first and last five codons of each CDS were omitted from the analysis of TEs. The Custom $\mathrm{R}$ scripts will be available upon requests.

\section{Bioinformatics and Computational Analyses}

639 Principal component analysis. To calculate the codon frequencies of individual genes from $\mathrm{H}$. 640 sapiens, we first downloaded coding sequences (CDS) data (Human genes, GRCh38p12) from the 641 Ensembl Biomart Database. For each CDS, we tabulated the occurrences of each codon - sans the 642 stop codons. We then expressed the codon counts as a percentage of the total number of codons in 643 its CDS to obtain the codon frequencies for each CDS. The codon frequencies for all 9666 CDS were 644 used as the input for the PCA using the Python 3.4 environment via the factoextra program [90].

645 Finally, the data was trimmed to remove truncated sequences as well as sequences with noncanonical start codons to a final of 9898 genes. 
647 Hierarchical clustering analysis. mRNA transcripts ranked in order of their half-lives, divided

648 equally into 4 groups and their average half-lives within each group was calculated. The

649 corresponding codon frequencies of transcripts within each group were averaged. Hierarchical

650 clustering was performed using the average linkage method to cluster the codon frequencies in $R$

651 using the ggplot2 program [91].

652 Quantification of GC3-content. To quantify GC3-content, we summed up the codon frequencies of

653 GC3 codons and expressed the frequencies on a percentage scale.

654 Calculation of CAl and CSC. cAl values were calculated using the standalone CAICal program [92]

655 in which the human mean codon usage dataset obtained from the Kazusa Codon Usage Database

656 [93] was used as the reference set. The CSC was calculated as described by Presnyak and

657 colleagues [14] using the HEK293 mRNA stability dataset (GSE69153) [Data ref: 31,32].

658 Binning of ribosomal occupancy frequencies and calculation of codon bias-derived occupancy

659 scores. To quantify codon bias for ribosome profiling, the factor loading scores of the codons from

660 the first principal component were normalized linearly on a percentage scale from 0 to 1 where 0

661 corresponded to the codon with the lowest score (AAT) and 1 for the codon with the highest score

662 (GCC) (Fig EV2A). Binning of the ribosome occupancies were performed in the R environment via a

663 custom script. To calculate the corresponding codon bias-derived occupancy scores, we substituted

664 the codon sequences of mRNA transcripts with their respective codon scores and in a similar fashion,

665 binned the data into 25 bins. As the scores of codons should inversely reflect the ribosome occupancy

666 (i.e. higher ribosome occupancy associated with lower codon scores), we calculated the reciprocal of

667 the binned codon scores within each bin for all 25 bins to derive the codon bias-derived occupancy

668 scores. Both ribosome occupancy and codon bias-derived occupancy scores were normalized on a

669 linear scale and a Pearson correlation performed on each transcript. To exclude the possibility that

670 the correlations were due to chance, we shuffled the bins for the codon bias-derived occupancy

671 scores within each individual transcripts and calculated the Pearson correlation between shuffled and

672 ribosomal occupancy data.

673 De novo motif discovery. Common transcripts which were more than 5-fold differentially upregulated

674 between the RIP-seq data [Data ref: 43,44 ] in JJN3 and H929 cells were firstly identified. The

675 corresponding cDNA sequences of the transcripts were downloaded from the UCSC table browser,

676 with the option of masking repeats in the sequences [94]. The sequences were subject to de novo

677 motif discovery via the MEME (Multiple EM for Motif Elicitation) software under the MEME tools suite

678 of programs [46].

679 Data Availability

680 Ribosome profiling and RNA-Seq results of HEK293 cells have been deposited at GEO and can be 681 accessed under dataset GSE126298.

\section{Acknowledgements}

683 The authors express their gratitude to all members of the laboratory of Medical Chemistry, Kyoto 684 University, for their kind advice and discussions. DNA libraries were sequenced by the Vincent J. 
685

686

687

688

689

690

691

692

693

694

695

696

697

698

699

700

701

702

703

704

705

706

707

708

709

710

711

712

713

714

715

716

717

718

Coates Genomics Sequencing Laboratory at UC Berkeley, supported by NIH S10 OD018174 Instrumentation Grant. Computations were supported by Manabu Ishii, Itoshi Nikaido, and the Bioinformatics Analysis Environment Service on RIKEN Cloud at RIKEN ACCC.

This work was supported by the JSPS KAKENHI (18H05278), AMED-CREST from Japan Agency for Medical Research and Development and the JSPS through Core-to-Core Program.

This work was supported by Joint Usage/Research Center program of Institute for Frontier Life and Medical Sciences, Takeda Science Foundation, the Uehara Memorial Foundation.

S.I. was supported by Grant-in-Aid for Scientific Research on Innovative Areas "nascent chain biology" (JP17H05679) and Grant-in-Aid for Young Scientists (A) (JP17H04998) from JSPS, the Pioneering Projects ("Cellular Evolution") and the Aging Project from RIKEN, and Takeda Science Foundation.

\section{Author contributions}

FH wrote the manuscript; together with SFY performed the experiments and analyzed the data. MY provided insightful comments and proofreading for the manuscript. YM and ML performed the mRNA decay experiments. YS, SI performed the ribosomal profiling and proofreading of the manuscript.SA and TN performed the ISRIM experiments. AV provided advice and bioinformatics expertise. AF and TF performed the polysome profiling experiments. OT supervised and designed the experiments.

\section{Conflict of interest}

The authors declare no conflict of interests.

\section{References}

1. Huang L, Lou C-H, Chan W, Shum EY, Shao A, Stone E, Karam R, Song H-W, Wilkinson MF (2011) RNA Homeostasis Governed by Cell Type-Specific and Branched Feedback Loops Acting on NMD. Molecular Cell 43: 950-961.

2. Mino T, Murakawa Y, Fukao A, Vandenbon A, Wessels H-H, Ori D, Uehata T, Tartey S, Akira S, Suzuki Y, et al. (2015) Regnase-1 and Roquin Regulate a Common Element in Inflammatory mRNAs by Spatiotemporally Distinct Mechanisms. Cell 161: 1058-1073.

3. Yoshinaga M, Nakatsuka Y, Vandenbon A, Ori D, Uehata T, Tsujimura T, Suzuki Y, Mino T, Takeuchi O (2017) Regnase-1 Maintains Iron Homeostasis via the Degradation of Transferrin Receptor 1 and Prolyl-Hydroxylase-Domain-Containing Protein 3 mRNAs. Cell Rep 19: 16141630.

4. Leppek K, Das R, Barna M (2018) Functional 5' UTR mRNA structures in eukaryotic translation regulation and how to find them. Nat Rev Mol Cell Biol 19: 158-174.

5. Cheng J, Maier KC, Avsec Ž, Rus P, Gagneur J (2017) Cis-regulatory elements explain most of the mRNA stability variation across genes in yeast. RNA 23: 1648-1659. 
6. Vogel C, Marcotte EM (2012) Insights into the regulation of protein abundance from proteomic and transcriptomic analyses. Nat Rev Genet 13: 227-232.

7. Zhou T, Weems M, Wilke CO (2009) Translationally Optimal Codons Associate with Structurally Sensitive Sites in Proteins. Mol Biol Evol 26: 1571-1580.

8. Sharp PM, Li WH (1987) The codon Adaptation Index--a measure of directional synonymous codon usage bias, and its potential applications. Nucleic Acids Res 15: 1281-1295.

9. Reis M dos, Savva R, Wernisch $L$ (2004) Solving the riddle of codon usage preferences: a test for translational selection. Nucleic Acids Res 32: 5036-5044.

10. dos Reis M, Wernisch L, Savva R (2003) Unexpected correlations between gene expression and codon usage bias from microarray data for the whole Escherichia coli K-12 genome. Nucleic Acids Res 31: 6976-6985.

11. Pechmann S, Frydman J (2013) Evolutionary conservation of codon optimality reveals hidden signatures of co-translational folding. Nat Struct Mol Biol 20: 237-243.

12. Dana A, Tuller $\mathrm{T}$ (2014) Mean of the typical decoding rates: a new translation efficiency index based on the analysis of ribosome profiling data. G3 (Bethesda) 5: 73-80.

13. Sabi R, Volvovitch Daniel R, Tuller T (2017) stAlcalc: tRNA adaptation index calculator based on species-specific weights. Bioinformatics 33: 589-591.

14. Presnyak V, Alhusaini N, Chen Y-H, Martin S, Morris N, Kline N, Olson S, Weinberg D, Baker KE, Graveley BR, et al. (2015) Codon optimality is a major determinant of mRNA stability. Cell 160: 1111-1124.

15. Radhakrishnan A, Chen Y-H, Martin S, Alhusaini N, Green R, Coller J (2016) The DEAD-Box Protein Dhh1p Couples mRNA Decay and Translation by Monitoring Codon Optimality. Cell 167: 122-132.e9.

16. Sweet T, Kovalak C, Coller J (2012) The DEAD-box protein Dhh1 promotes decapping by slowing ribosome movement. PLOS Biol 10: e1001342.

17. Dana A, Tuller T (2014) The effect of tRNA levels on decoding times of mRNA codons. Nucleic Acids Res 42: 9171-9181.

18. Gardin J, Yeasmin R, Yurovsky A, Cai Y, Skiena S, Futcher B Measurement of average decoding rates of the 61 sense codons in vivo. eLife 3 :.

19. Drummond DA, Wilke CO (2008) Mistranslation-induced protein misfolding as a dominant constraint on coding-sequence evolution. Cell 134: 341-352.

20. Akashi H (1994) Synonymous Codon Usage in Drosophila Melanogaster: Natural Selection and Translational Accuracy. Genetics 136: 927-935.

21. Harigaya $Y$, Parker R (2016) Analysis of the association between codon optimality and mRNA stability in Schizosaccharomyces pombe. BMC Genomics 17: 895.

22. Lee Y, Zhou T, Tartaglia GG, Vendruscolo M, Wilke CO (2010) Translationally optimal codons associate with aggregation-prone sites in proteins. Proteomics 10: 4163-4171.

23. Mishima Y, Tomari Y (2016) Codon Usage and 3' UTR Length Determine Maternal mRNA Stability in Zebrafish. Molecular Cell 61: 874-885. 
24. Burow DA, Martin S, Quail JF, Alhusaini N, Coller J, Cleary MD (2018) Attenuated Codon Optimality Contributes to Neural-Specific mRNA Decay in Drosophila. Cell Rep 24: 1704-1712.

25. Boël G, Letso R, Neely H, Price WN, Wong K-H, Su M, Luff J, Valecha M, Everett JK, Acton TB, et al. (2016) Codon influence on protein expression in E. coli correlates with mRNA levels. Nature 529: 358-363.

26. Jeacock L, Faria J, Horn D (2018) Codon usage bias controls mRNA and protein abundance in trypanosomatids. Elife 7:.

27. de Freitas Nascimento J, Kelly S, Sunter J, Carrington M (2018) Codon choice directs constitutive mRNA levels in trypanosomes. Elife 7:.

28. Wu Q, Medina SG, Kushawah G, DeVore ML, Castellano LA, Hand JM, Wright M, Bazzini AA (2019) Translation affects mRNA stability in a codon-dependent manner in human cells. Elife 8:.

29. Forrest ME, Narula A, Sweet TJ, Arango D, Hanson G, Ellis J, Oberdoerffer S, Coller J, Rissland OS (2018) Codon usage and amino acid identity are major determinants of mRNA stability in humans. bioRxiv 488676.

30. Zerbino DR, Achuthan P, Akanni W, Amode MR, Barrell D, Bhai J, Billis K, Cummins C, Gall A, Girón CG, et al. (2018) Ensembl 2018. Nucleic Acids Res 46: D754-D761.

31. Murakawa Y, Hinz M, Mothes J, Schuetz A, Uhl M, Wyler E, Yasuda T, Mastrobuoni G, Friedel CC, Dölken L, et al. (2015) Gene Expression Omnibus GSE69153 (https://www.ncbi.nlm.nih.gov/geo/query/acc.cgi?acc=GSE69153) . [DATASET]

32. Murakawa Y, Hinz M, Mothes J, Schuetz A, Uhl M, Wyler E, Yasuda T, Mastrobuoni G, Friedel CC, Dölken L, et al. (2015) RC3H1 post-transcriptionally regulates A20 mRNA and modulates the activity of the IKK/NF-KB pathway. Nat Commun 6: 7367.

33. Bazzini AA, Del Viso F, Moreno-Mateos MA, Johnstone TG, Vejnar CE, Qin Y, Yao J, Khokha MK, Giraldez AJ (2016) Codon identity regulates mRNA stability and translation efficiency during the maternal-to-zygotic transition. EMBO J 35: 2087-2103.

34. McGlincy NJ, Ingolia NT (2017) Transcriptome-wide measurement of translation by ribosome profiling. Methods 126: 112-129.

35. Tuller T, Kupiec M, Ruppin E (2007) Determinants of Protein Abundance and Translation Efficiency in S. cerevisiae. PLOS Computational Biology 3: e248.

36. Iwasaki S, Ingolia NT (2016) Seeing translation. Science 352: 1391-1392.

37. Ingolia NT, Ghaemmaghami S, Newman JRS, Weissman JS (2009) Genome-Wide Analysis in Vivo of Translation with Nucleotide Resolution Using Ribosome Profiling. Science 324: 218-223.

38. Duncan CDS, Mata J (2017) Effects of cycloheximide on the interpretation of ribosome profiling experiments in Schizosaccharomyces pombe. Sci Rep 7: 1-11.

39. Gerashchenko MV, Gladyshev VN (2014) Translation inhibitors cause abnormalities in ribosome profiling experiments. Nucleic Acids Res 42: e134.

40. Santos DA, Shi L, Tu BP, Weissman JS (2019) Cycloheximide can distort measurements of mRNA levels and translation efficiency. Nucleic Acids Res 47: 4974-4985. 
41. Adachi S, Homoto M, Tanaka R, Hioki Y, Murakami H, Suga H, Matsumoto M, Nakayama KI, Hatta T, lemura S, et al. (2014) ZFP36L1 and ZFP36L2 control LDLR mRNA stability via the ERK-RSK pathway. Nucleic Acids Res 42: 10037-10049.

42. Kuwano Y, Pullmann R, Marasa BS, Abdelmohsen K, Lee EK, Yang X, Martindale JL, Zhan M, Gorospe M (2010) NF90 selectively represses the translation of target mRNAs bearing an AUrich signature motif. Nucleic Acids Res 38: 225-238.

43. Marchesini M, Ogoti Y, Fiorini E, Aktas Samur A, Nezi L, D'Anca M, Storti P, Samur MK, GananGomez I, Fulciniti MT, et al. (2017) Gene Expression Omnibus GSE83662 (https://www.ncbi.nlm.nih.gov/geo/query/acc.cgi?acc=GSE83662). [DATASET]

44. Marchesini M, Ogoti Y, Fiorini E, Aktas Samur A, Nezi L, D'Anca M, Storti P, Samur MK, GananGomez I, Fulciniti MT, et al. (2017) ILF2 Is a Regulator of RNA Splicing and DNA Damage Response in 1q21-Amplified Multiple Myeloma. Cancer Cell 32: 88-100.e6.

45. Snyder M (2017) ENCODE Project Experiment ENCSR073QLQ (https://www.encodeproject.org/experiments/ENCSR073QLQ/) [DATASET]

46. Bailey TL, Boden M, Buske FA, Frith M, Grant CE, Clementi L, Ren J, Li WW, Noble WS (2009) MEME SUITE: tools for motif discovery and searching. Nucleic Acids Res 37: W202-208.

47. Dotu I, Adamson SI, Coleman B, Fournier C, Ricart-Altimiras E, Eyras E, Chuang JH (2018) SARNAclust: Semi-automatic detection of RNA protein binding motifs from immunoprecipitation data. PLoS Comput Biol 14: e1006078.

48. Zheng G, Qin Y, Clark WC, Dai Q, Yi C, He C, Lambowitz AM, Pan T (2015) Efficient and quantitative high-throughput transfer RNA sequencing. Nat Methods 12: 835-837.

49. Gingold H, Tehler D, Christoffersen NR, Nielsen MM, Asmar F, Kooistra SM, Christophersen NS, Christensen LL, Borre M, Sørensen KD, et al. (2014) A Dual Program for Translation Regulation in Cellular Proliferation and Differentiation. Cell 158: 1281-1292.

50. Charneski CA, Hurst LD (2013) Positively charged residues are the major determinants of ribosomal velocity. PLoS Biol 11: e1001508.

51. Ingolia NT, Lareau LF, Weissman JS (2011) Ribosome profiling of mouse embryonic stem cells reveals the complexity and dynamics of mammalian proteomes. Cell 147: 789-802.

52. Artieri CG, Fraser HB (2014) Accounting for biases in riboprofiling data indicates a major role for proline in stalling translation. Genome Res 24: 2011-2021.

53. Lareau LF, Hite DH, Hogan GJ, Brown PO (2014) Distinct stages of the translation elongation cycle revealed by sequencing ribosome-protected mRNA fragments. Elife 3: e01257.

54. Pop C, Rouskin S, Ingolia NT, Han L, Phizicky EM, Weissman JS, Koller D (2014) Causal signals between codon bias, mRNA structure, and the efficiency of translation and elongation. Mol Syst Biol 10: 770.

55. Endoh T, Sugimoto $N(2016)$ Mechanical insights into ribosomal progression overcoming RNA G-quadruplex from periodical translation suppression in cells. Sci Rep 6: 22719.

56. Thandapani P, Song J, Gandin V, Cai Y, Rouleau SG, Garant J-M, Boisvert F-M, Yu Z, Perreault J-P, Topisirovic I, et al. (2015) Aven recognition of RNA G-quadruplexes regulates translation of the mixed lineage leukemia protooncogenes. Elife 4:. 
57. Pan L, Li Y, Zhang H-Y, Zheng Y, Liu X-L, Hu Z, Wang Y, Wang J, Cai Y-H, Liu Q, et al. (2017) DHX15 is associated with poor prognosis in acute myeloid leukemia (AML) and regulates cell apoptosis via the NF-kB signaling pathway. Oncotarget 8: 89643-89654.

58. Faure G, Ogurtsov AY, Shabalina SA, Koonin EV (2016) Role of mRNA structure in the control of protein folding. Nucleic Acids Res 44: 10898-10911.

59. Yu C-H, Dang Y, Zhou Z, Wu C, Zhao F, Sachs MS, Liu Y (2015) Codon Usage Influences the Local Rate of Translation Elongation to Regulate Co-translational Protein Folding. Mol Cell 59: 744-754.

60. Tuller T, Veksler-Lublinsky I, Gazit N, Kupiec M, Ruppin E, Ziv-Ukelson M (2011) Composite effects of gene determinants on the translation speed and density of ribosomes. Genome Biol 12: R110.

61. Edri S, Tuller T (2014) Quantifying the effect of ribosomal density on mRNA stability. PLoS ONE 9: e102308.

62. Neymotin B, Ettorre V, Gresham D (2016) Multiple Transcript Properties Related to Translation Affect mRNA Degradation Rates in Saccharomyces cerevisiae. G3 (Bethesda) 6: 3475-3483.

63. Schwartz DC, Parker $R$ (2000) mRNA decapping in yeast requires dissociation of the cap binding protein, eukaryotic translation initiation factor 4E. Mol Cell Biol 20: 7933-7942.

64. Chan LY, Mugler CF, Heinrich S, Vallotton P, Weis K (2018) Non-invasive measurement of mRNA decay reveals translation initiation as the major determinant of mRNA stability. Elife 7:.

65. Schwartz DC, Parker R (1999) Mutations in translation initiation factors lead to increased rates of deadenylation and decapping of mRNAs in Saccharomyces cerevisiae. Mol Cell Biol 19: 5247-5256.

66. Saini P, Eyler DE, Green R, Dever TE (2009) Hypusine-containing protein elF5A promotes translation elongation. Nature 459: 118-121.

67. Zhou Z, Dang Y, Zhou M, Li L, Yu C, Fu J, Chen S, Liu Y (2016) Codon usage is an important determinant of gene expression levels largely through its effects on transcription. Proc Natl Acad Sci U S A 113: E6117-E6125.

68. Zhou Z, Dang Y, Zhou M, Yuan H, Liu Y (2018) Codon usage biases co-evolve with transcription termination machinery to suppress premature cleavage and polyadenylation. Elife 7:.

69. Kudla G, Lipinski L, Caffin F, Helwak A, Zylicz M (2006) High Guanine and Cytosine Content Increases mRNA Levels in Mammalian Cells. PLoS Biol 4:.

70. Newman ZR, Young JM, Ingolia NT, Barton GM (2016) Differences in codon bias and GC content contribute to the balanced expression of TLR7 and TLR9. Proc Natl Acad Sci USA 113: E1362-1371.

71. Fu J, Dang Y, Counter C, Liu Y (2018) Codon usage regulates human KRAS expression at both transcriptional and translational levels. J Biol Chem 293: 17929-17940.

72. Lampson BL, Pershing NLK, Prinz JA, Lacsina JR, Marzluff WF, Nicchitta CV, MacAlpine DM, Counter CM (2013) Rare codons regulate KRas oncogenesis. Curr Biol 23: 70-75. 
73. Freimer JW, Hu TJ, Blelloch R (2018) Decoupling the impact of microRNAs on translational repression versus RNA degradation in embryonic stem cells. Elife 7:

74. Grinberg-Bleyer $\mathrm{Y}$, Oh H, Desrichard A, Bhatt DM, Caron R, Chan TA, Schmid RM, Hayden MS, Klein U, Ghosh S (2017) NF-kB c-Rel Is Crucial for the Regulatory T Cell Immune Checkpoint in Cancer. Cell 170: 1096-1108.e13.

75. Oh H, Grinberg-Bleyer Y, Liao W, Maloney D, Wang P, Wu Z, Wang J, Bhatt DM, Heise N, Schmid RM, et al. (2017) An NF-kB Transcription-Factor-Dependent Lineage-Specific Transcriptional Program Promotes Regulatory T Cell Identity and Function. Immunity 47: 450465.e5.

76. Gumienny R, Zavolan M (2015) Accurate transcriptome-wide prediction of microRNA targets and small interfering RNA off-targets with MIRZA-G. Nucleic Acids Res 43: 9095.

77. Zafrir Z, Tuller $T$ (2015) Nucleotide sequence composition adjacent to intronic splice sites improves splicing efficiency via its effect on pre-mRNA local folding in fungi. RNA 21: 17041718.

78. Amit M, Donyo M, Hollander D, Goren A, Kim E, Gelfman S, Lev-Maor G, Burstein D, Schwartz S, Postolsky B, et al. (2012) Differential GC content between exons and introns establishes distinct strategies of splice-site recognition. Cell Rep 1: 543-556.

79. Zhang J, Kuo CCJ, Chen L (2011) GC content around splice sites affects splicing through premRNA secondary structures. BMC Genomics 12: 90.

80. Parrott AM, Walsh MR, Mathews MB (2007) Analysis of RNA:protein interactions in vivo: identification of RNA-binding partners of nuclear factor 90. Meth Enzymol 429: 243-260.

81. Zhou Z, Licklider LJ, Gygi SP, Reed R (2002) Comprehensive proteomic analysis of the human spliceosome. Nature 419: 182-185.

82. Pfeifer I, Elsby R, Fernandez M, Faria PA, Nussenzveig DR, Lossos IS, Fontoura BMA, Martin WD, Barber GN (2008) NFAR-1 and -2 modulate translation and are required for efficient host defense. Proc Natl Acad Sci USA 105: 4173-4178.

83. Guan D, Altan-Bonnet N, Parrott AM, Arrigo CJ, Li Q, Khaleduzzaman M, Li H, Lee C-G, Pe'ery $\mathrm{T}$, Mathews MB (2008) Nuclear factor 45 (NF45) is a regulatory subunit of complexes with NF90/110 involved in mitotic control. Mol Cell Biol 28: 4629-4641.

84. Harashima A, Guettouche T, Barber GN (2010) Phosphorylation of the NFAR proteins by the dsRNA-dependent protein kinase PKR constitutes a novel mechanism of translational regulation and cellular defense. Genes Dev 24: 2640-2653.

85. Wolkowicz UM, Cook AG (2012) NF45 dimerizes with NF90, Zfr and SPNR via a conserved domain that has a nucleotidyltransferase fold. Nucleic Acids Res 40: 9356-9368.

86. Graber T, Baird S, Kao P, Mathews M, Holcik M (2010) NF45 functions as an IRES trans-acting factor that is required for translation of clAP1 during the unfolded protein response. Cell Death Differ 17: 719-729.

87. Gratacós FM, Brewer G (2010) The role of AUF1 in regulated mRNA decay. Wiley Interdiscip Rev RNA 1: 457-473. 
88. Akichika S, Hirano S, Shichino Y, Suzuki T, Nishimasu H, Ishitani R, Sugita A, Hirose Y, Iwasaki S, Nureki O, et al. (2019) Cap-specific terminal N6-methylation of RNA by an RNA polymerase II-associated methyltransferase. Science 363:

89. Anders S, Huber W (2010) Differential expression analysis for sequence count data. Genome Biology 11: R106.

90. Kassambara A, Mundt F (2017) factoextra: Extract and Visualize the Results of Multivariate Data Analyses.

91. Wickham H, Chang W, Henry L, Pedersen TL, Takahashi K, Wilke C, Woo K, RStudio (2018) ggplot2: Create Elegant Data Visualisations Using the Grammar of Graphics.

92. Puigbò P, Bravo IG, Garcia-Vallve S (2008) CAlcal: a combined set of tools to assess codon usage adaptation. Biol Direct 3: 38.

93. Nakamura Y, Gojobori T, Ikemura T (2000) Codon usage tabulated from international DNA sequence databases: status for the year 2000. Nucleic Acids Res 28: 292.

94. Karolchik D, Hinrichs AS, Furey TS, Roskin KM, Sugnet CW, Haussler D, Kent WJ (2004) The UCSC Table Browser data retrieval tool. Nucleic Acids Res 32: D493-496.

\section{TABLE AND FIGURES LEGENDS}

Figure 1. Bioinformatics analysis reveals that biased codons can be categorized into GC3 and AT3 codons respectively.

A. Hierarchical clustering analysis of model organisms and their average CDS codon frequencies.

B. Principal component analysis of the CDS codon frequencies of 9666 protein-coding genes. PC1 and PC2 indicate the first and second principal components.

C. Heatmap of half-lives of mRNA and their CDS codon frequencies. The transcripts were ranked according to their half-lives and divided equally into quartiles. The respective codon frequencies of each group were then averaged.

D. Histogram illustrating the distribution of genes and their respective GC3- and GC-content.

E. Comparison of average transcript mRNA half-lives across their respective GC3- and GC-content ranges. Number of transcripts within each gene optimality range is indicated above their respective points.

Data information: In (E), error bars represent the $95 \%$ confidence intervals.

Figure 2. GC3-AT3 codon bias can explain ribosome occupancy to a certain extent

A. Average ribosome occupancy and their respective codon bias-derived occupancy scores across the CDS of transcript (in 25 bins). Ribosome occupancy for 16,423 transcripts and their respective 
947 codon bias-derived occupancy were firstly binned into 25 bins and the mean occupancy was

948 calculated for each bin.

949 B. Cumulative distribution plots showing the distributions of correlations between ribosome occupancy

950 and codon bias-derived occupancy. Correlations obtained from the ribosome occupancy and

951 scrambled codon bias-derived occupancy served as the control. A Kolmogorov-Smirnov test was

952 performed between the codon bias-derived occupancy and the control group.

953 C. Comparison of average transcript translation efficiencies (TEs) across their respective GC3-content 954 ranges. Number of transcripts within each gene optimality range is indicated above their respective 955 points.

956 D, E. Principal component analysis of CDS codon frequencies of protein-coding genes derived from a

$957+1$ frameshift (D) and a -1 frameshift (E). Shaded ellipses indicate codons which are GC-rich (orange) 958 and AT-rich (blue).

959 Data information: In (A, C), error bars represent the 95\% confidence intervals.

\section{Figure 3. GC3-content of transcripts determine their fate.}

961 A. HEK293 Tet-off experiments showing the degradation of REL-OPT and REL-WT transcripts (left)

962 as well as IL6-OPT, IL6-WT and IL6-DE transcripts (right), post-doxycycline addition.

963 B. Representative immunoblot of FLAG-tagged REL-OPT and REL-WT in HEK293T cells transfected 964 with either empty plasmids, plasmids bearing REL-OPT or REL-WT. The immunoblot is representative 965 of 3 independent experiments. ACTB is shown as the loading controls.

966 C. ELISA of secreted IL6 concentrations of IL6-OPT, IL6-WT and IL6-DE from HEK293T cells

967 transfected with plasmids bearing IL6-OPT, IL6-WT and IL6-DE.

968 D. Fold changes of REL-OPT and REL-WT transcript levels (top) relative to their abundances from 969 fraction 1 as detected by GPCR across polysome fractions (below). Data represents the mean \pm SD 970 for 3 biological replicates.

971 Data information: In (A, D), data is representative of 3 independent experiments each with 3

972 replicates. The data represents the mean \pm SD for 3 replicates. A two-way ANOVA with Holm-Sidak 973 multiple comparisons was performed. P-values are denoted as follows: $p<0.05\left(^{*}\right), p<0.01\left(^{* *}\right)$ and $\left.974 \mathrm{p}<0.001{ }^{* * \star}\right)$. The half-lives of the respective transcripts are indicated in brackets. In (C), the data is 975 representative of 3 independent experiments each with 3 replicates. The data represents the mean \pm 976 SD for 3 replicates. A one-way ANOVA with Tukey's multiple comparisons was performed between 977 samples where, $\left.p<0.01{ }^{* *}\right)$ and $\left.p<0.0011^{(* *}\right)$.

\section{Figure 4. GC-content as an additional determinant of stability}


A, B. HEK293 Tet-off experiments showing the degradation of REL-OPT and REL-WT transcripts (A) and IL6-OPT, IL6-WT and IL6-DE transcripts (B), under vehicle (DMSO)- and cycloheximide (CHX)treatment, post-doxycycline addition.

C, D. HEK293 Tet-off experiments showing the degradation of REL-OPT and REL-WT transcripts (C) and IL6-OPT, IL6-WT and IL6-DE transcripts (D), under vehicle (PBS)- and anisomycin (ANI)treatment, post-doxycycline addition.

E, F. HEK293 Tet-off experiments showing the degradation of REL-OPT, REL-OPT (+1 Frameshift) and REL-WT transcripts (E) as well as IL6-OPT, IL6-WT, IL6-DE and IL6-OPT (+1 Frameshift) transcripts (F) post-doxycycline addition.

In (A-F), data is representative of 3 independent experiments each with 3 replicates. The data represents the mean \pm SD for 3 replicates. A two-way ANOVA with Holm-Sidak multiple comparisons was performed. P-values are denoted as follows: $\left.p<0.05\left(^{*}\right), p<0.01{ }^{(* *}\right)$ and $\left.p<0.001{ }^{* \star *}\right)$.

Figure 5. RNA binding proteins bind differentially to transcripts with different levels of GC3content.

A, B, C. Volcano plots showing the enrichment of RBPs which bind to REL-WT relative to REL-OPT transcripts (A), IL6-DE relative to IL6-WT transcripts (B) and IL6-WT relative to IL6-OPT transcripts (C).

Data information: In (A, B, C), vertical dotted lines indicate a 1.5-fold enrichment while horizontal dashed lines indicate the $p$-value cut-off of 0.05 . Points shaded in blue indicate RBPs which have a differential fold change of more than 1.5 and $p<0.05$.

Figure 6. ILF2 regulates the stability of low GC3 / high AT3 transcripts

1000

A. Cumulative distribution plots showing the difference in distribution of transcript optimality between

1001 upregulated and downregulated transcripts in K562 cells subject to ILF2 CRISPR interference

1002 targeting ILF2. Transcript quantities are indicated in the figure legend.

1003

B, C. HEK293 Tet-off experiments showing the degradation of REL-OPT (B) and REL-WT (C) transcripts with ILF2 and ILF3 siRNA and Control (CTR) siRNA treatment, post-doxycycline addition.

D. Representative immunoblot of FLAG-tagged REL-OPT and REL-WT expressed in HEK293T cells under ILF2 and ILF3 siRNA treatment. The immunoblot is representative of 3 independent experiments. ACTB is shown as loading controls.

E. Representative immunoblot of FLAG-tagged REL-OPT and REL-WT in HEK293T cells coexpressed with two different isoforms of ILF2. The immunoblot is representative of 3 independent experiments. ACTB is shown as loading controls.

1011 Data information: In (A), Wilcoxon signed rank tests were performed on the upregulated and 
1013 representative of 3 independent experiments in which the data represents the mean \pm SD for 3

1014 biological replicates. A two-way ANOVA with Holm-Sidak multiple comparisons was performed. P-

1015 values are denoted as follows: $\left.p<0.05\left(^{*}\right), p<0.01{ }^{(*}\right)$.

1016

Expanded View Figure 1. Bioinformatics analysis reveals that GC3-content is a determinant of

1017 stabiity.

1018 A. Principal component analysis of the CDS codon frequencies of protein-coding genes in S.

1019 cerevisiae. PC1 and PC2 indicate the first and second principal components.

1020

B. PC1 factor loadings of codons from the yeast dataset ranked from the highest to the lowest. The optimal and non-optimal designation at the bottom of the figure refers to the designation according to Presnyak and colleagues [14].

1023

C. Pearson correlation between PC1 factor loading scores and CSC for individual codons (excluding stop codons).

1025

D. Pearson correlation between GC-content and GC3-content for 9666 protein-coding genes.

E, F. Violin plots (E) and cumulative relative frequency distributions $(\mathbf{F})$ visualizing the distribution of mRNA half-lives across their respective GC3-content brackets.

1028

G. Comparison of average transcript mRNA half-lives across their respective cAl. Number of transcripts within each range is indicated above their respective points.

1030

H. Gene ontology analysis (biological processes) of the top 5\% ranked genes in terms of gene GC3-

1031 content

1032 I. Gene ontology analysis (biological processes) of the bottom 5\% ranked genes in terms of gene

1033 GC3-content

1034 Data information: In (E), the box plots within each figure are indicative of the median and interquartile ranges. In $(\mathbf{F})$, Wilcoxon signed rank tests were performed on the various distributions against the control (All transcripts) group. P-values are denoted. In (G), error bars represent the 95\% confidence intervals.

1038 Expanded View Figure 2. GC3-content can explain ribosome occupancy and translation 1039 efficiency to a certain extent

1040 A. PC1 factor loadings of codons from the human dataset ranked from the highest to the lowest

1041 (bottom) and their corresponding normalized factor loadings after linear normalization onto a 1042 percentage scale (top).

B. Pearson correlation between the correlations of derived from comparison of ribosome occupancy and codon bias-derived scores for two ribosome profiling sample replicates. 
C. Pearson correlation between codon bias-derived occupancy scores and ribosome occupancy for individual codons (excluding stop codons).

D. Three example transcripts (EIF2B2, DYNC1LI2 and IDH3G) which demonstrate high correlation between ribosome occupancy and codon bias-derived scores (left) as well as their corresponding Pearson correlations over 25 bins (right).

1050

E. Comparison of average transcript translation efficiencies (TEs) across their respective GC3-content

1051 ranges after grouping by mRNA abundances. Error bars represent the $95 \%$ confidence intervals.

F, G. Hierarchical clustering analysis of half-lives of mRNA and their CDS codon frequencies after a +1 frameshift $(\mathbf{F})$ and -1 frameshift $(\mathbf{G})$. The transcripts were ranked according to their half-lives and divided equally into quartiles. The respective codon frequencies of each group were then averaged. Codon highlights indicate codons which are GC-rich (yellow) and AT-rich (blue).

Expanded View Figure 3. GC3-content of transcripts affects translation efficiency and stability

A. Example of how transcript GC3-optimization and deoptimization was performed to generate GC3optimized and de-optimized versions of $R E L$ and IL6 transcripts.

1059

B. GC3- and GC-content of REL-OPT/WT, REL-OPT (+1 Frameshift), as well as IL6-OPT/WT/DE and IL6-OPT (+1 Frameshift) transcripts.

1061

C. Protein abundance of immunoblot of FLAG-tagged REL-OPT and REL-WT in HEK293T cells (normalized by respective mRNA levels) transfected with either empty plasmids, plasmids bearing REL-OPT or REL-WT (corresponding to Fig 3B). The data is representative of 3 independent experiments. The respective steady state mRNA levels (transcript copy numbers) are shown on the right.

D. IL6 Protein abundance as determined by ELISA of IL6-OPT, IL6-WT and IL6-DE in HEK293T cells (normalized by respective mRNA levels) transfected with either empty plasmids, plasmids bearing IL6-OPT, IL6-WT or IL6-DE (corresponding to Fig 3C). The ELISA quantification is representative of 3 independent experiments. The respective steady state mRNA levels (transcript copy numbers) are shown on the right.

E. Representative immunoblot of FLAG-tagged REL-OPT and REL-WT expressed in HeLa cells (left).

1072 The immunoblot is representative of 3 independent experiments.

1073 F. mRNA stability experiments showing the degradation of REL-OPT and REL-WT transcripts in HeLa 1074 cells, post-actinomycin-D addition.

1075 Data information: In (C), the densitometry data is representative of 3 independent experiments.

1076 Unpaired t-tests were performed within the REL-OPT and REL-WT samples, $p<0.05\left({ }^{*}\right)$. In (D), a oneway ANOVA with Tukey's multiple comparisons was performed between samples where, $\left.p<0.01{ }^{* *}\right)$ and $\left.p<0.001{ }^{* * *}\right)$. In $(\mathbf{F})$, data is representative of 3 independent experiments each with 3 replicates. 
1079 The data represents the mean \pm SD for 3 replicates. A two-way ANOVA with Holm-Sidak multiple 1080 comparisons was performed. P-values are denoted as follows: $\left.p<0.001{ }^{* \star *}\right)$.

1081 Expanded View Figure 4. RNA binding proteins can be identified from ISRIM experiments

1082 A. Venn diagram indicating the number of RBPs identified from the IL6 ISRIM experiments.

1083

B. Venn diagram indicating the number of RBPs identified from the REL and IL6 ISRIM experiments.

1084 Expanded View Figure 5. ILF2 is an RNA binding protein that can bind differentially to 1085 transcripts with different levels of GC3-content.

1086 A. Cumulative distribution plots showing the GC3-content distribution of transcripts bound to by ILF2

1087 in H929 (top) and JJN3 cells (bottom). Wilcoxon signed rank tests were performed on the ILF2 RIP

1088 group against the control group. $\mathrm{P}$-values are denoted in the figure.

1089

B. Scatterplot of the RPKM values of mRNA transcripts in K562 cells subject to ILF2 CRISPR

1090 interference and its corresponding WT control. mRNA transcripts are colored according to their

1091 respective GC3-content.

1092

C. Fold changes of example mRNA representing low, average and high GC3-content transcripts from

1093 the RPKM values of mRNA transcripts in K562 cells subject to ILF2 CRISPR interference.

1094

D. Densitometric analysis of immunoblot of FLAG-tagged REL-OPT and REL-WT expressed in HEK293T cells under ILF2 and ILF3 siRNA treatment (corresponding to Fig 6D).

1096

E. Densitometric analysis of immunoblot of FLAG-tagged REL-OPT and REL-WT expressed in

1097 HEK293T cells co-expressed with two different isoforms of ILF2 (corresponding to Figure 6E)

1098 F-G. Top three RNA Motifs enriched in upregulated transcripts ( $>5$ fold) in ILF2 RIP-seq data (Fig

1099 EV5A) derived from both H929 and JJN3 datasets (left) and their corresponding annotations in 1100 transcripts (right) (F), followed by the ILF3 motif and its distribution identified by Dotu et al [47] from 1101 ILF3 RNA Bind-n-seq experiments (G).

1102 Data information: In (D, E), densitometry data is representative of 3 independent experiments. A 1103 one-way ANOVA with Tukey's multiple comparisons was performed within the REL-OPT and REL-WT 1104 samples. P-values are denoted as follows, $\left.p<0.05\left(^{*}\right), p<0.01{ }^{(*}\right)$ and $\left.p<0.001{ }^{* \star *}\right)$.

1105 Dataset EV1. List of genes and their corresponding GC3- and GC-content

1106 Dataset EV2. List of Sequences of synthesized constructs as well as qPCR primers and their 1107 corresponding sequences

1108 Dataset EV3. List of RBPs identified in ISRIM experiments 
Pigure 1

A
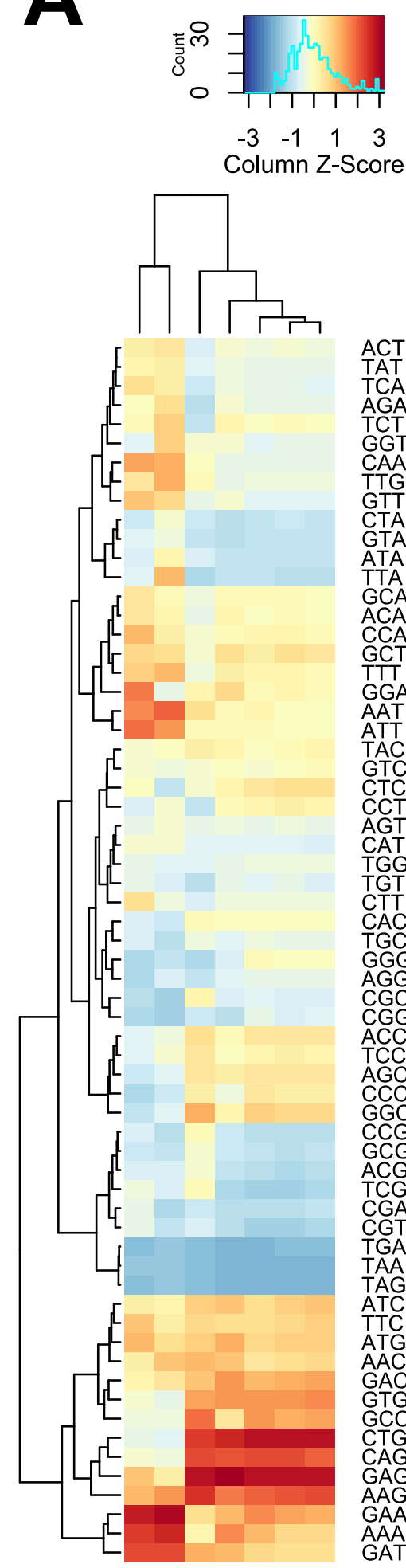

ACT
AAT
TCA
AGA
TCT
GGT
GAA
CAA
TTG
GT
CTA
GTA
ATA
TTA
GCA
ACA
CCA
GCT
ITT
GGA
AAT
ATT
TAC
GTC
CTC
CCT
AGT
CAT
TGG
TGT
CTT
CAC
TGC
GGG
AGG
CGC
CGG
ACC
TCC
AGC
CCC
GGC
CCG
GCG
ACG
TCG
CGA
CGT
TGA
TAA
TAG
ATC
TTC
ATG
AAC
GAC
GTG
GCC
CTG
CAG
GAG
AAG
GAA
AAA
GAT

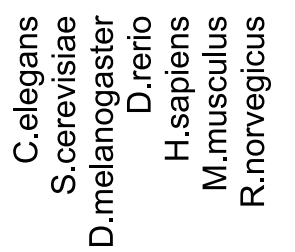

\section{C}
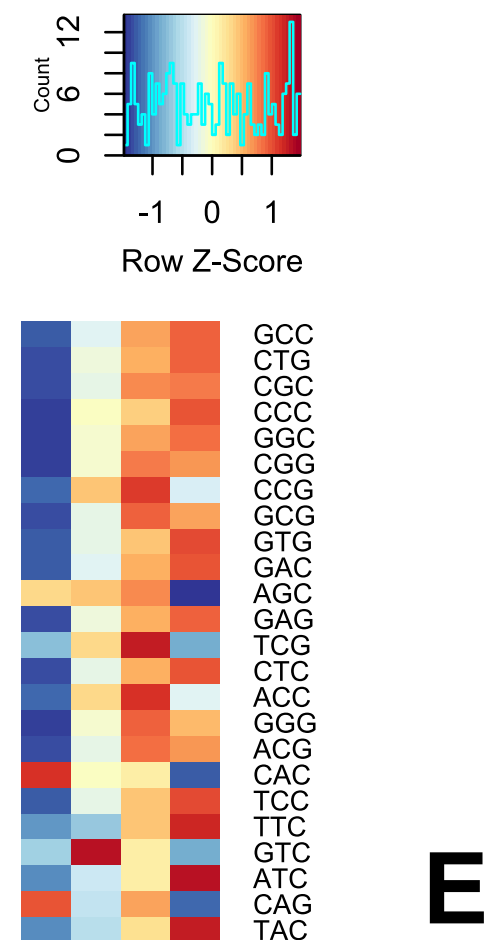

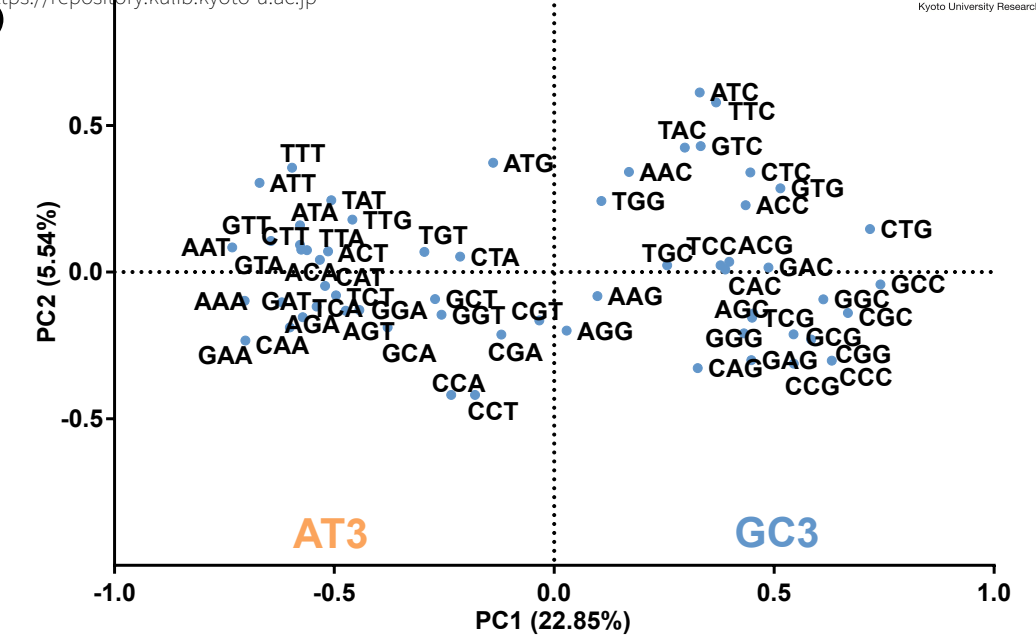

D
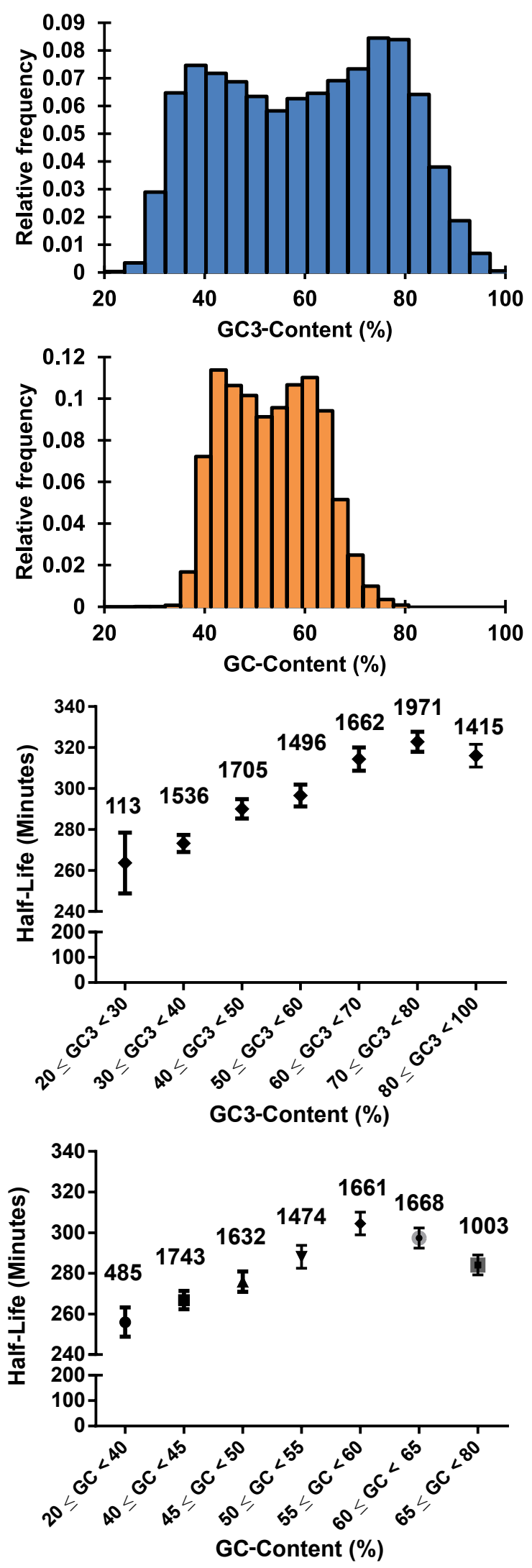

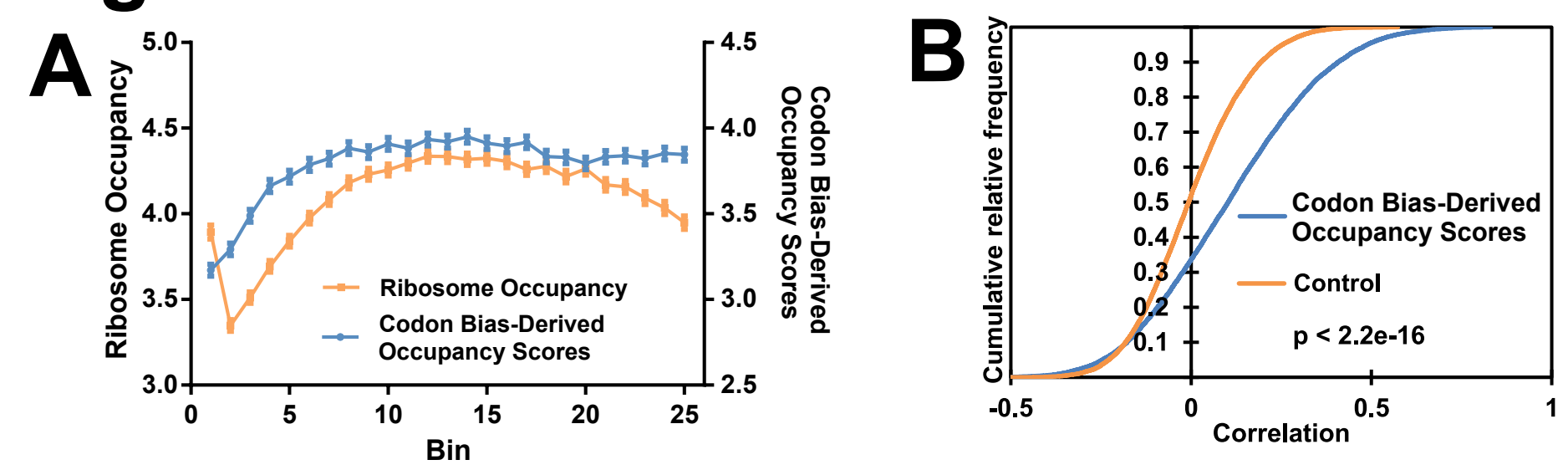

C

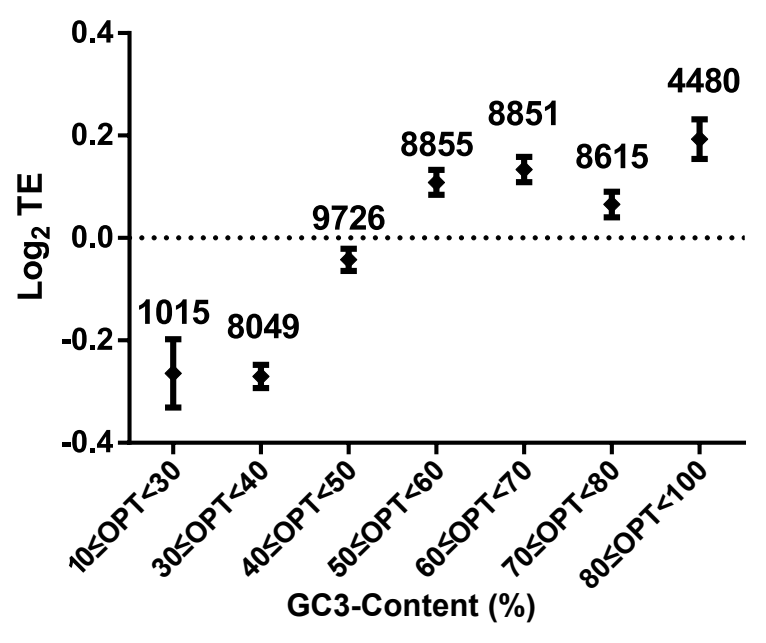

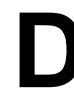

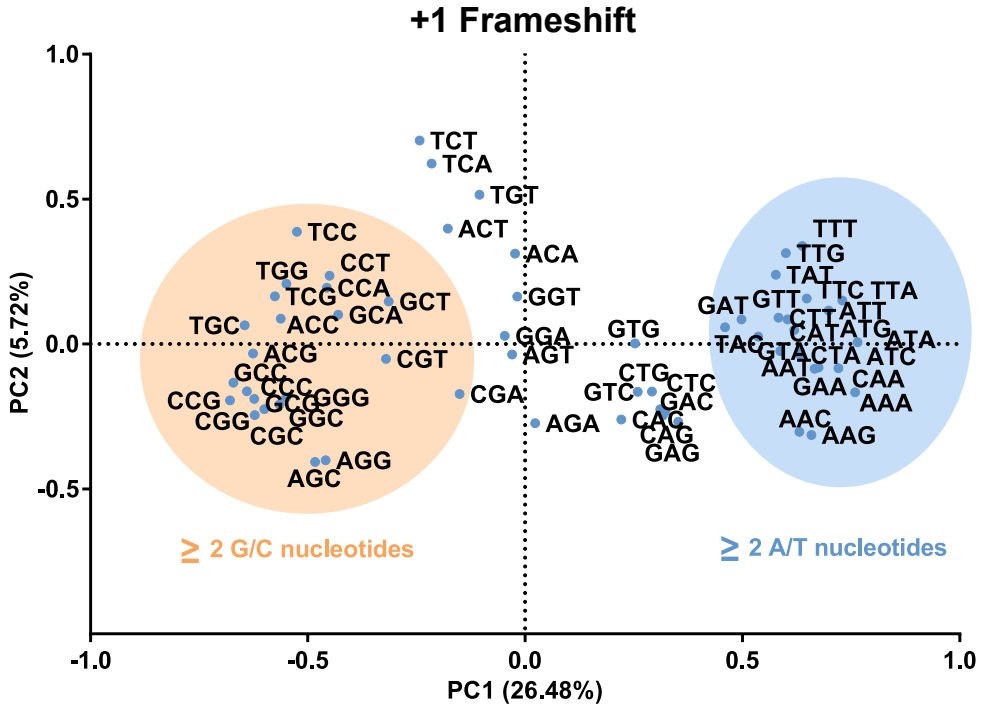

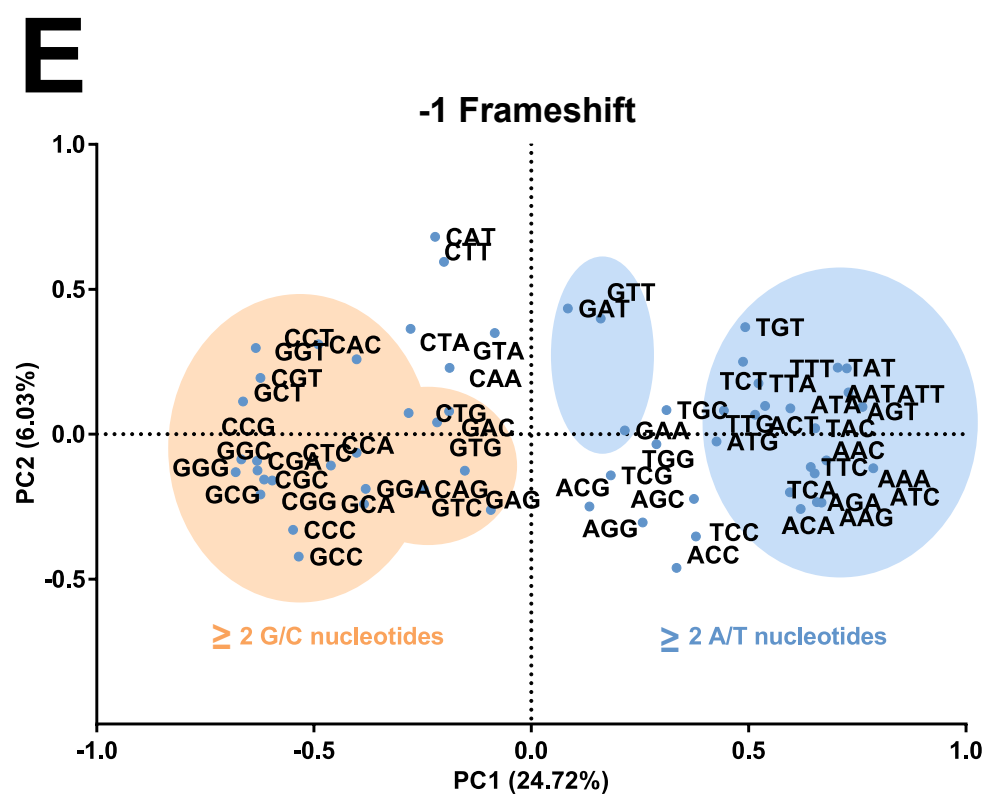



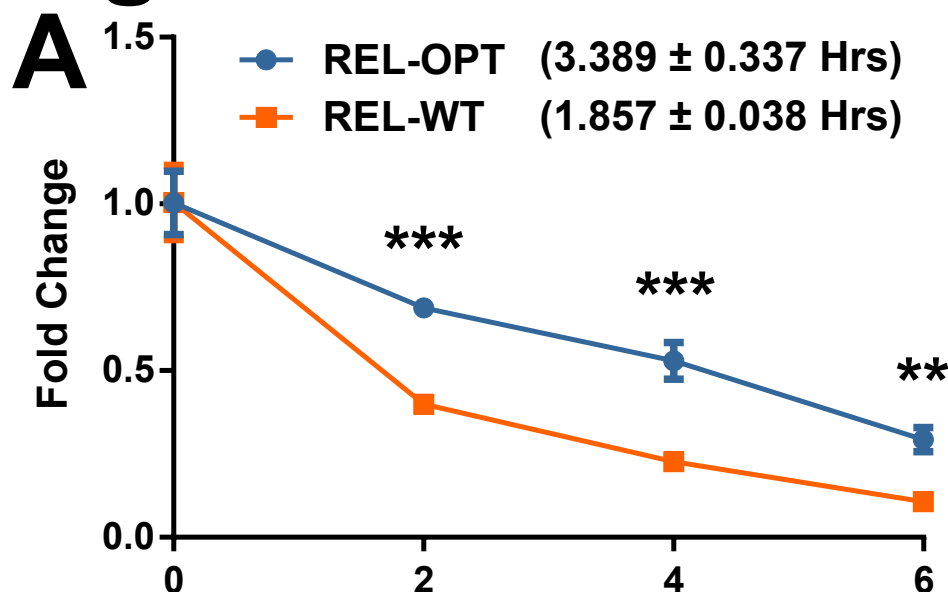

Time (Hours Post-Doxycycline Addition)

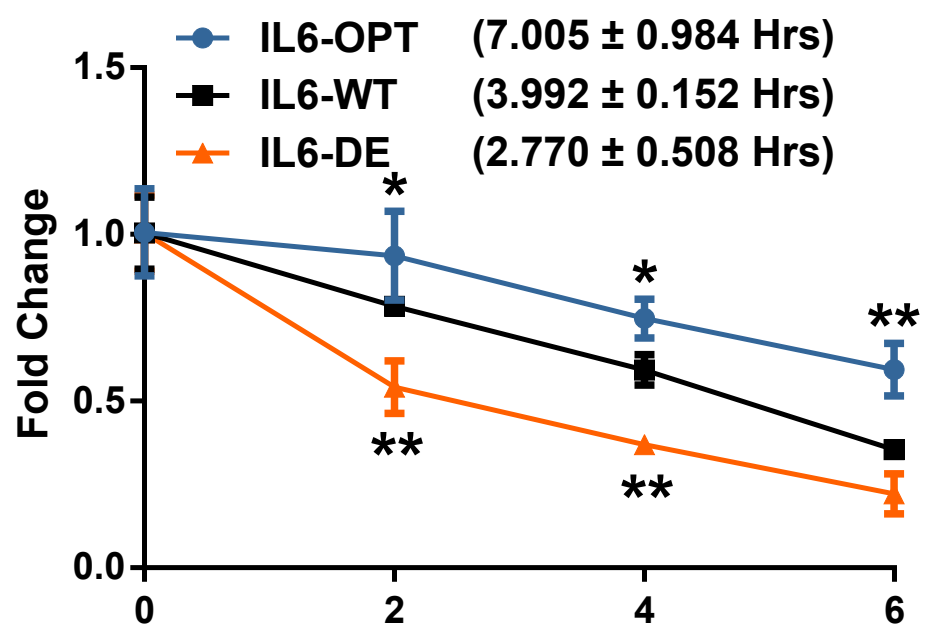

Time (Hours Post-Doxycycline Addition)

B

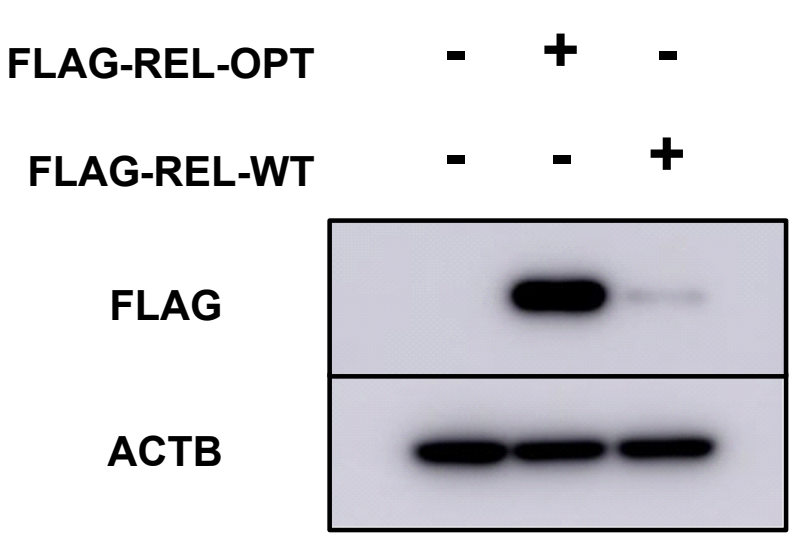

D

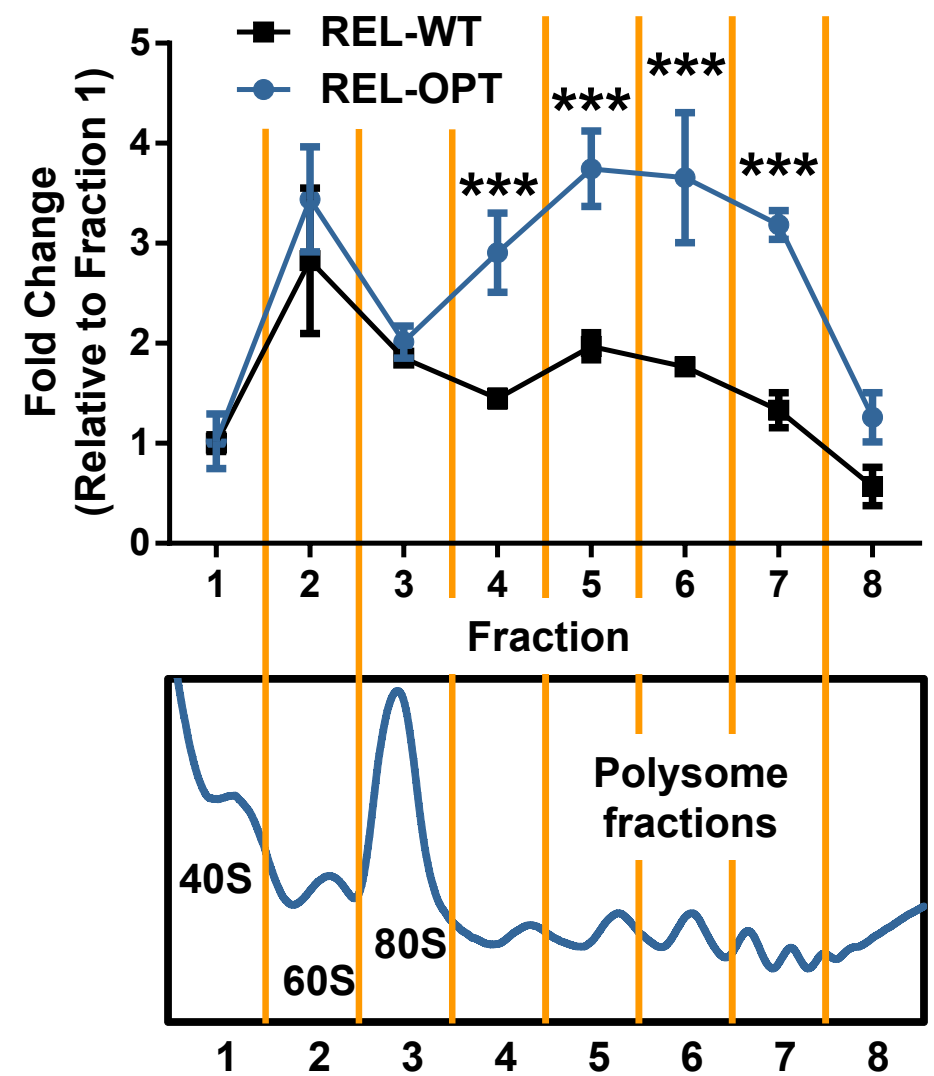


A
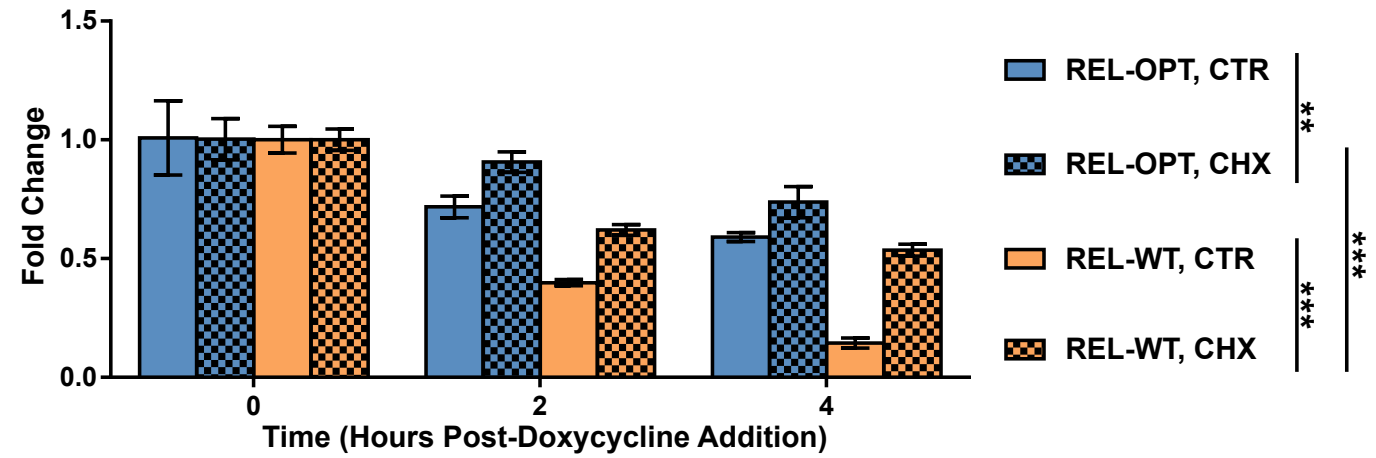

B
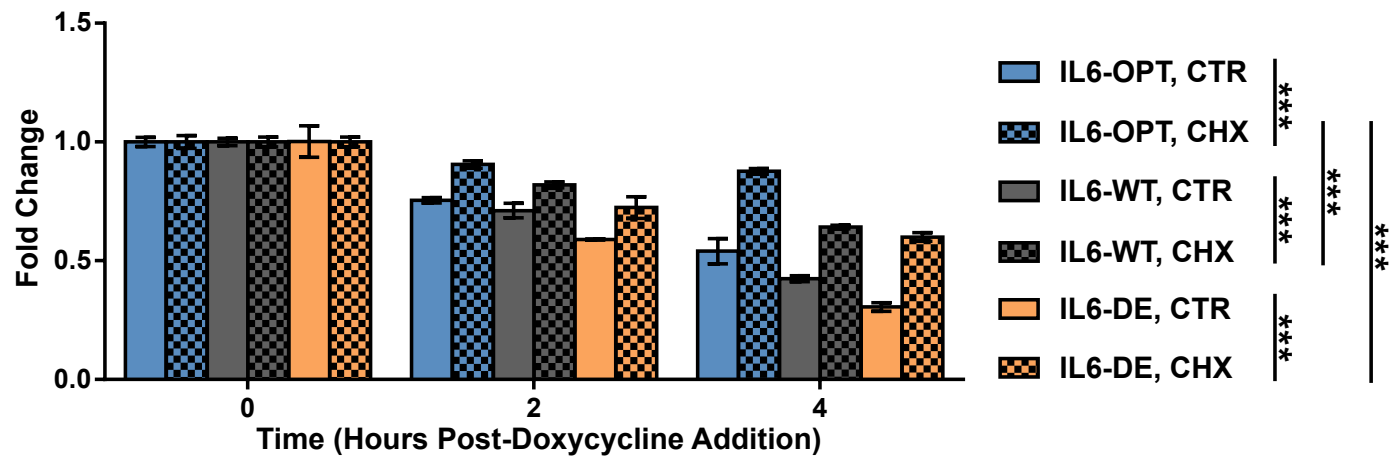

C
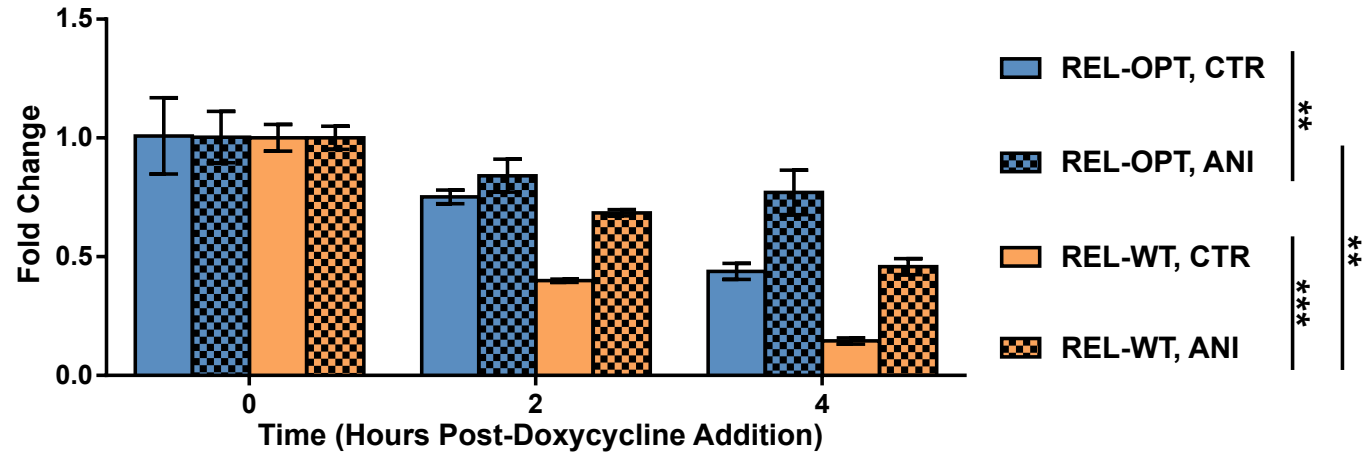

D
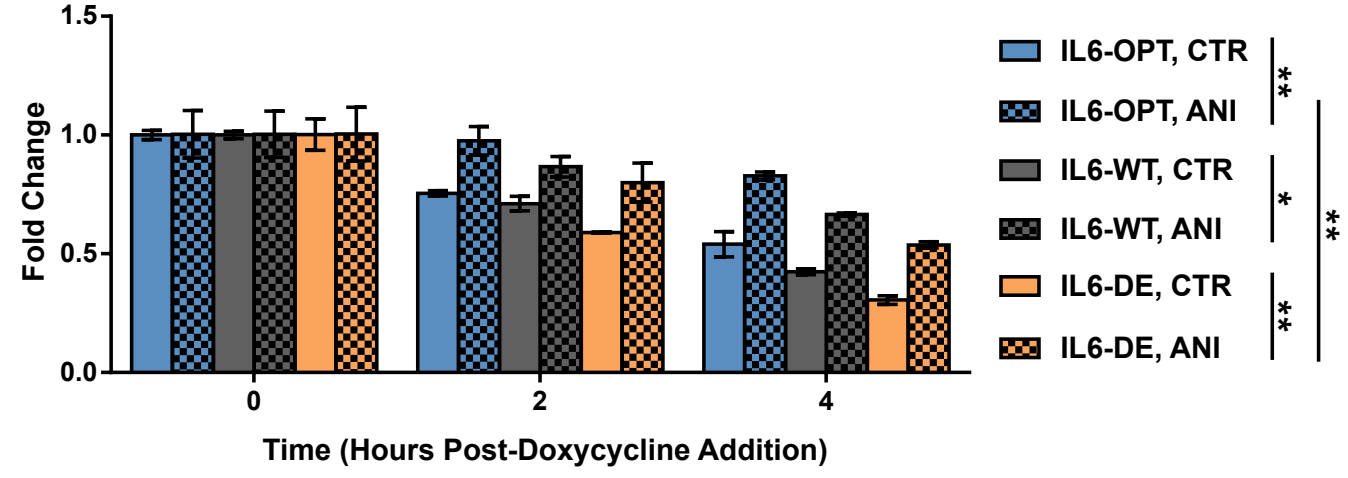

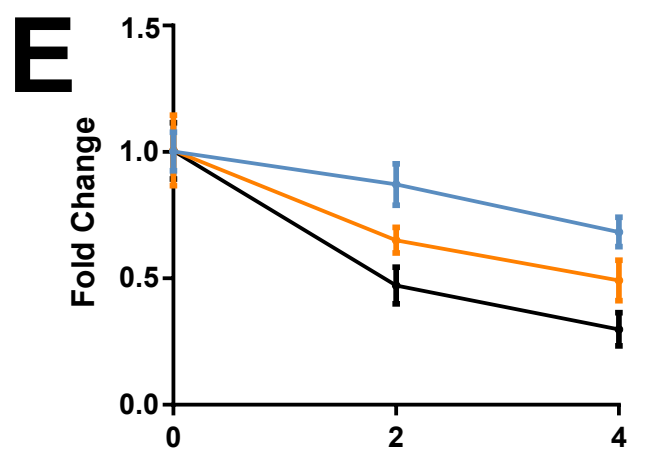

Time (Hours Post-Doxycycline Addition)

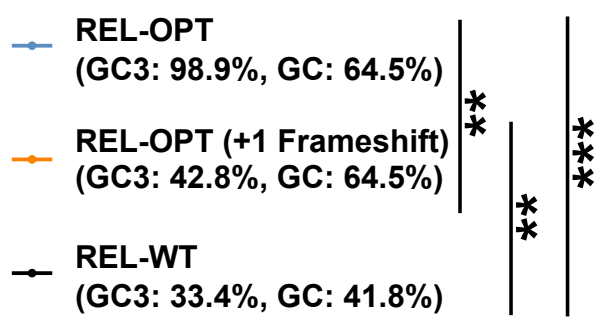

Time (Hours Post-Doxycycline Addition)

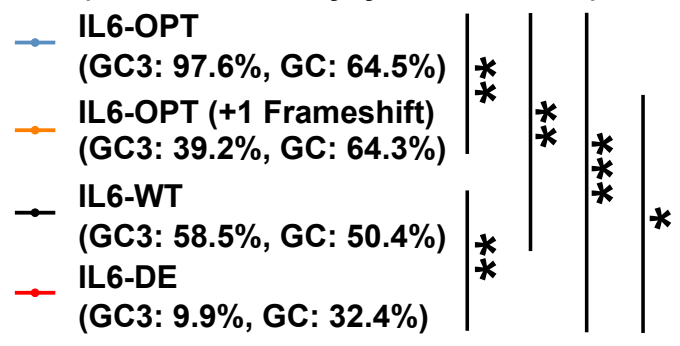



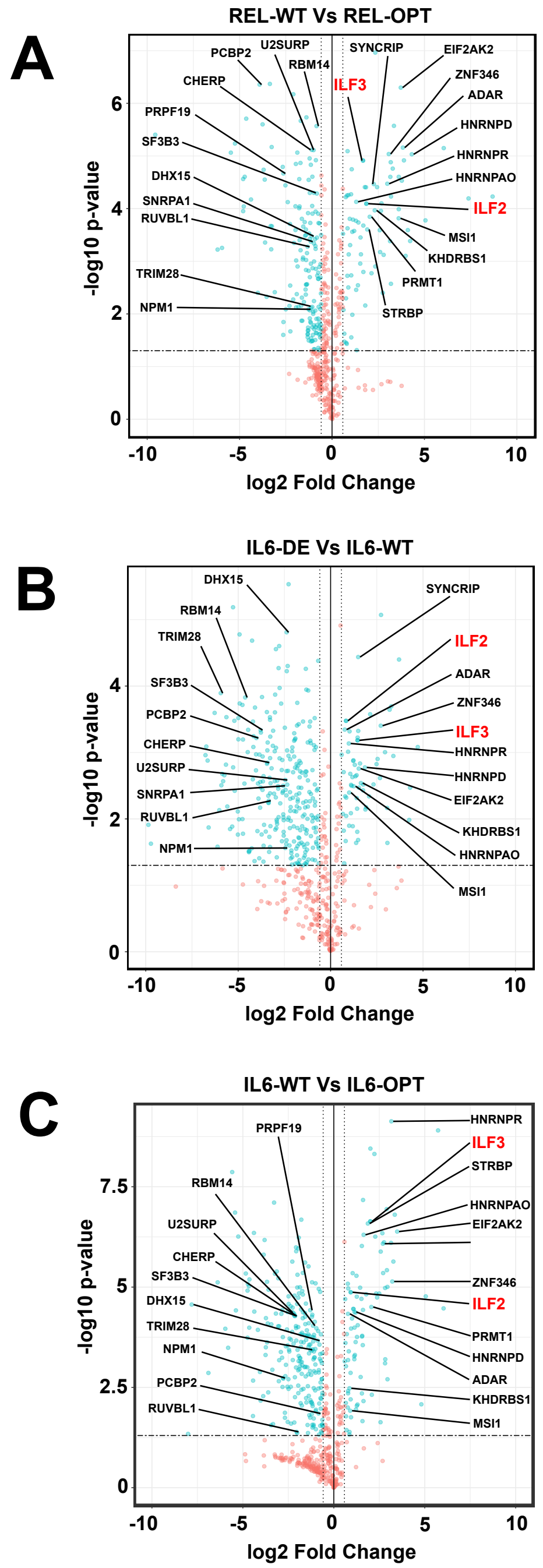
A

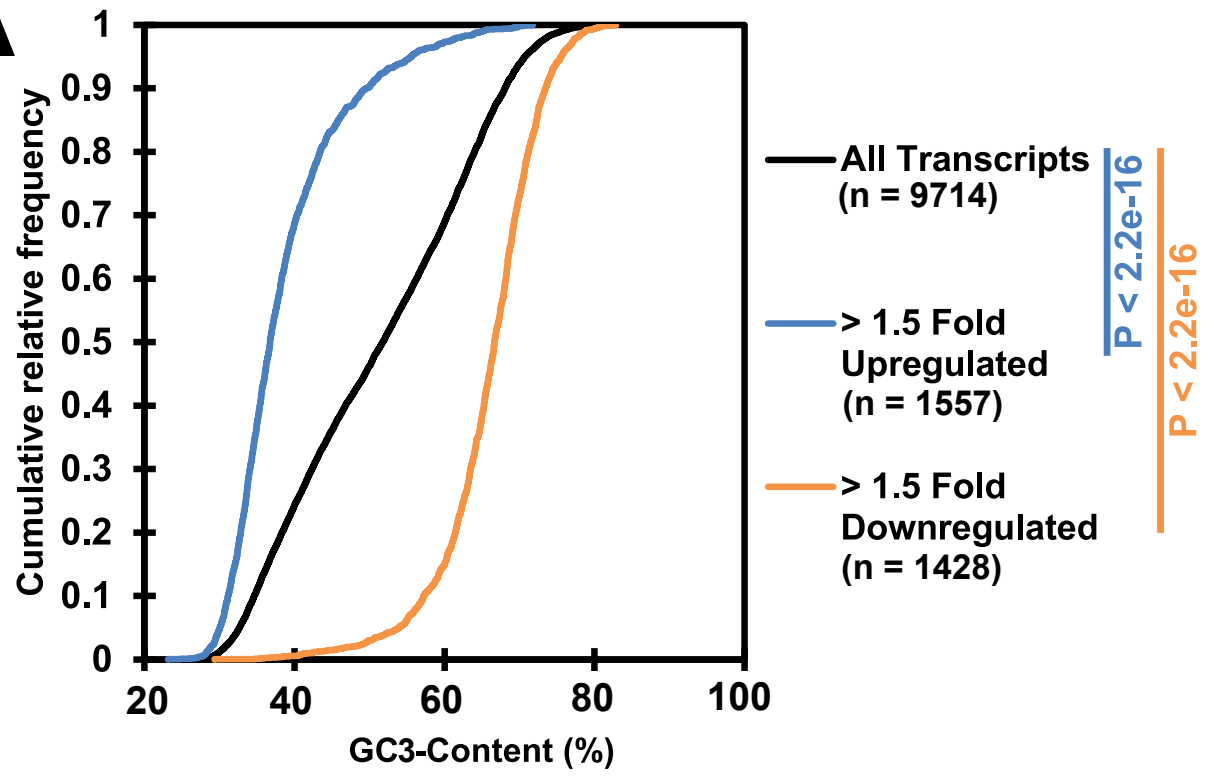

B

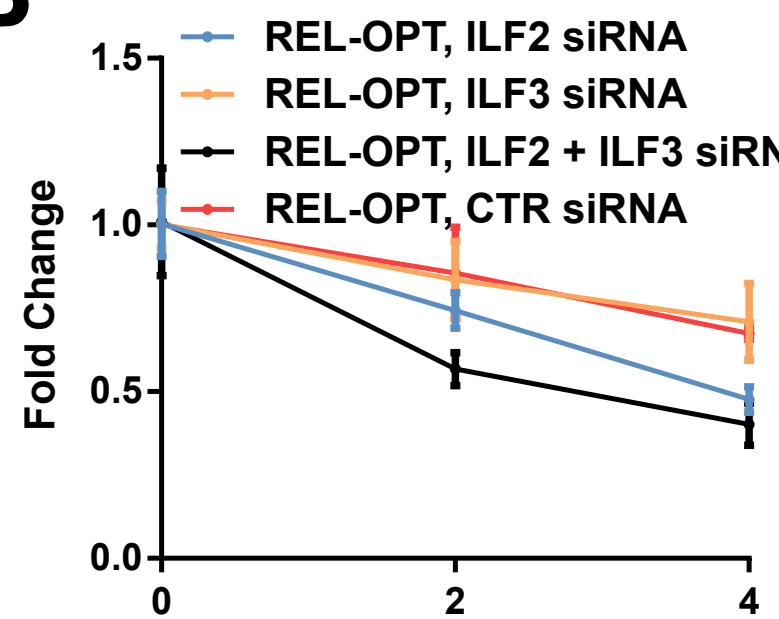

Time (Hours Post-Doxycycline Addition)

D

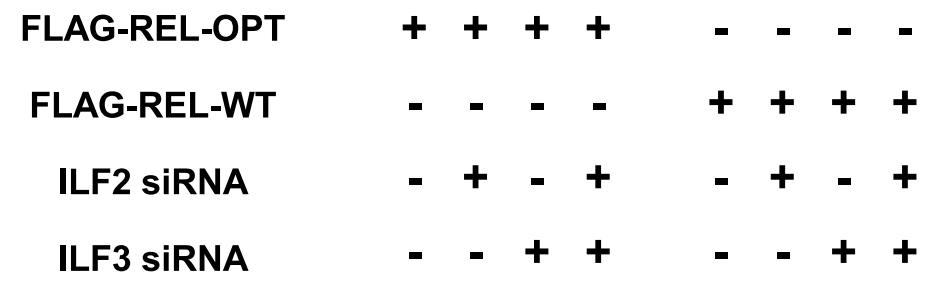

FLAG

(Short exposure)

FLAG

(Long exposure)

ACTB

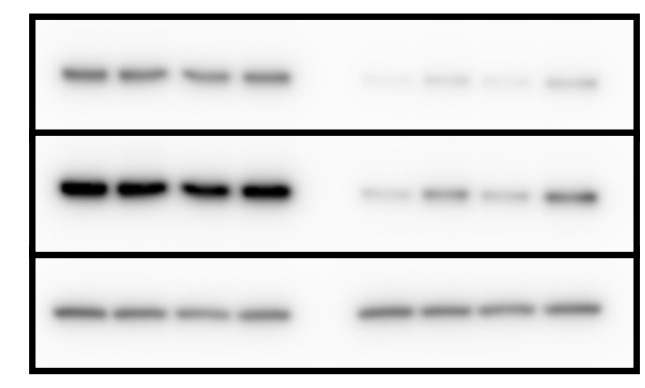

ILF2

ILF3

$\mid \begin{aligned} & \text { NF110 } \\ & \text { NF90 }\end{aligned}$

ACTB

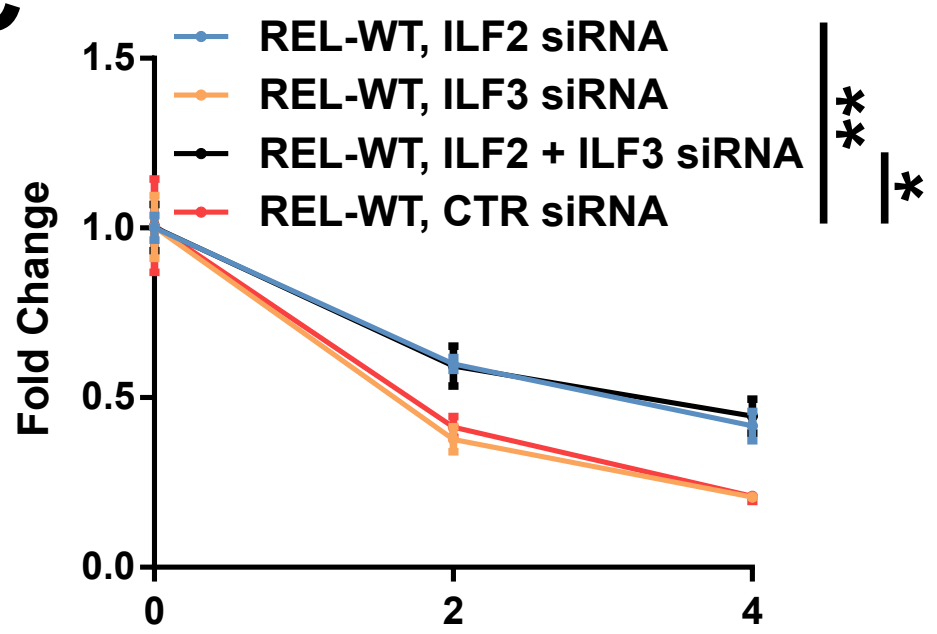

Time (Hours Post-Doxycycline Addition)

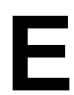

FLAG-REL-OPT $\quad+\quad+\quad+$

FLAG-REL-WT

ILF2 isoform 1

ILF2 isoform 2

FLAG

(short exposure)

FLAG

(long exposure)

АСTB

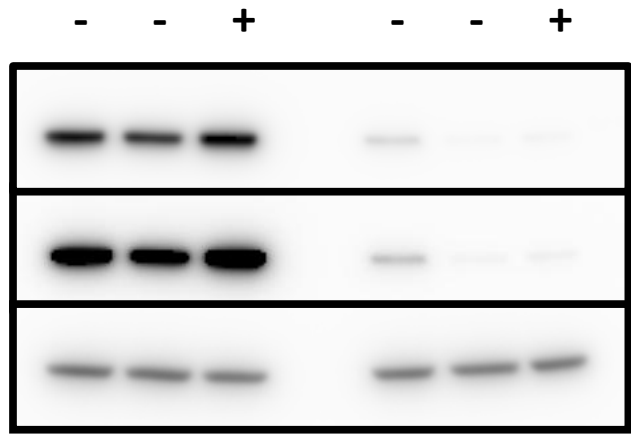

ILF2 Isoform 1 (45kD)

Isoform 2 (38kD)

ACTB 

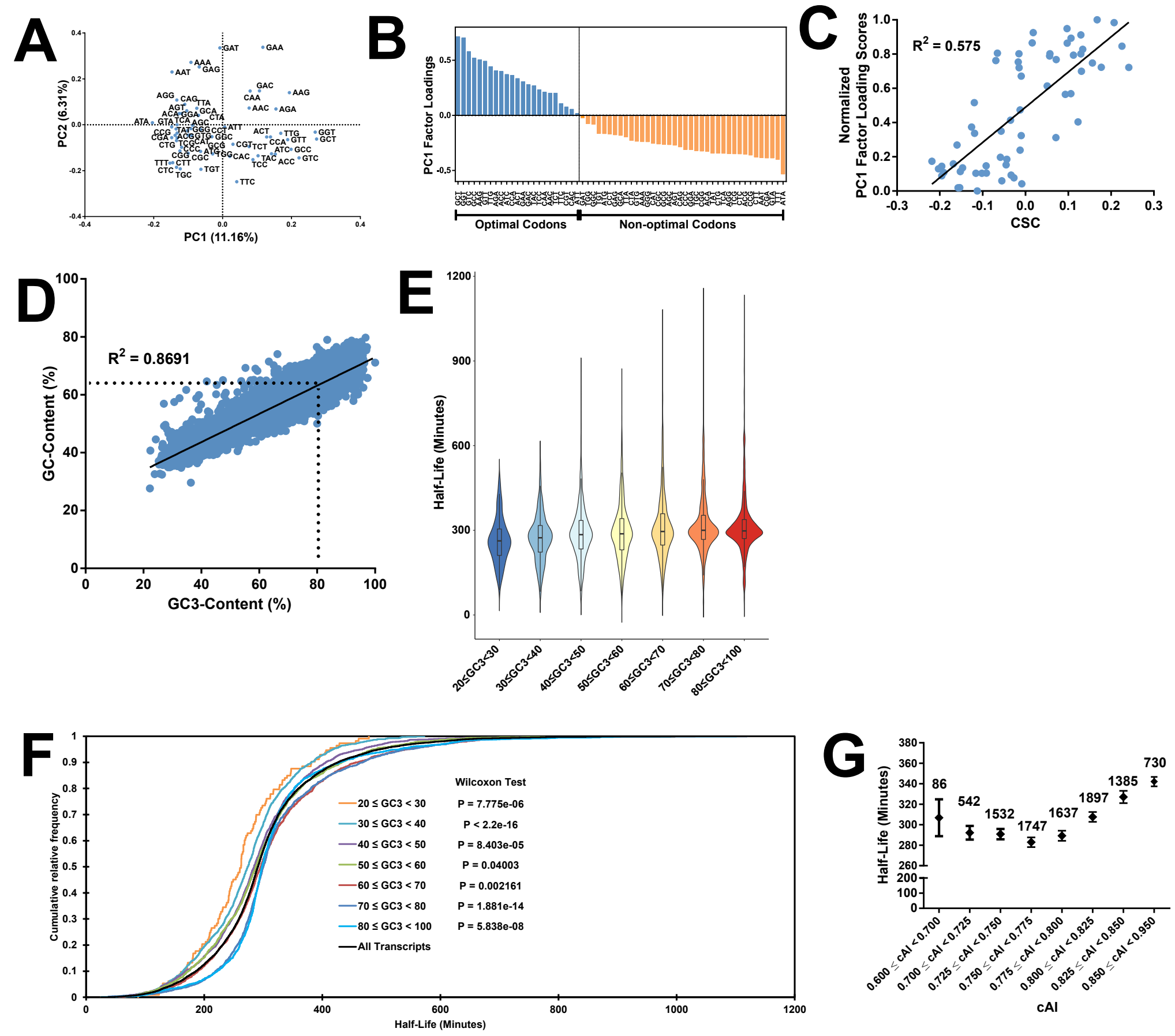

H

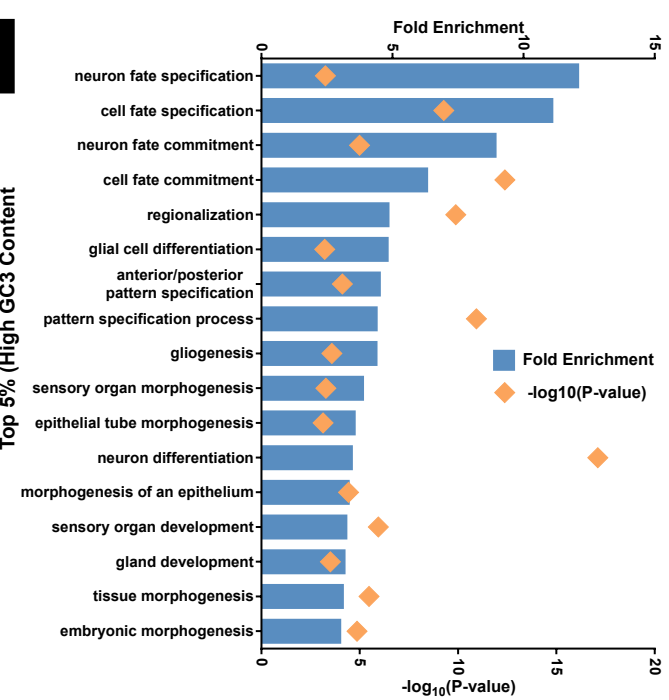

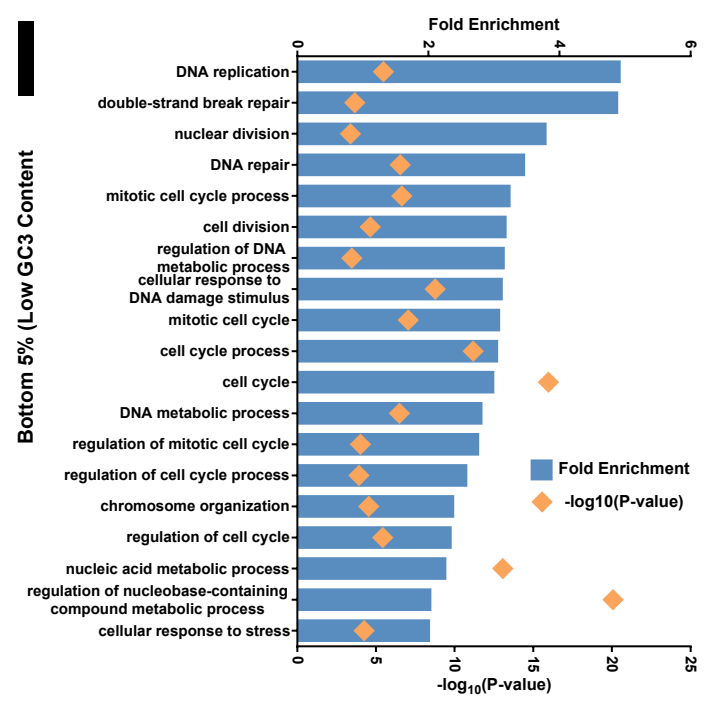




\section{2}

A

... GCC GCC GGC ...

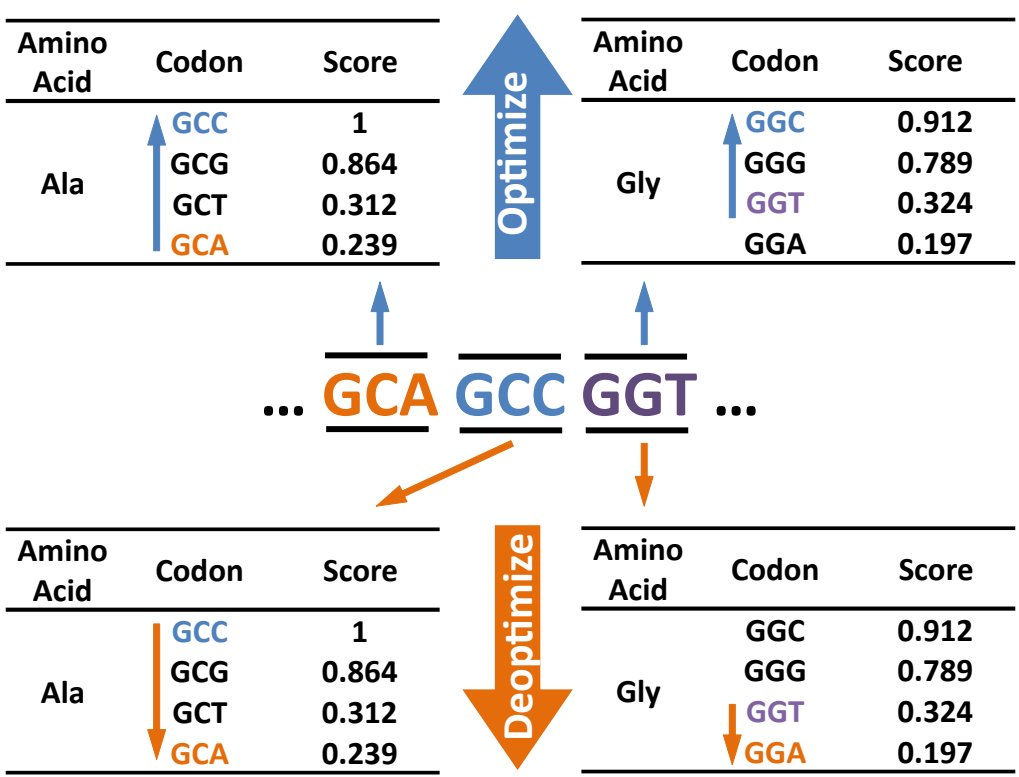

B

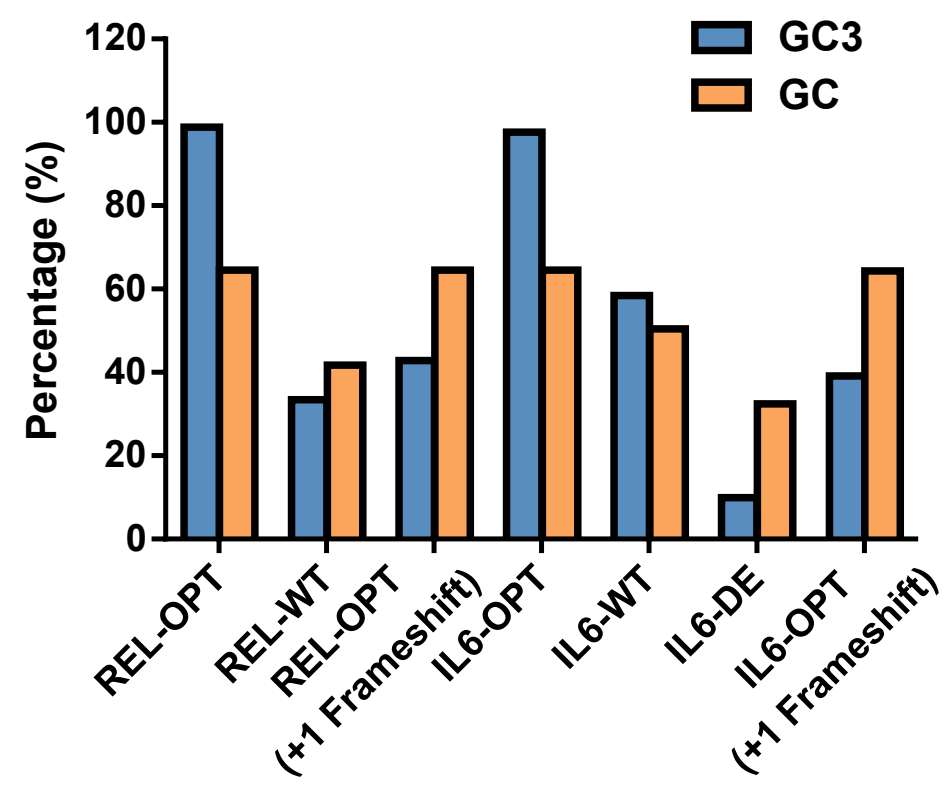

C

... GCA GCA GGA ...
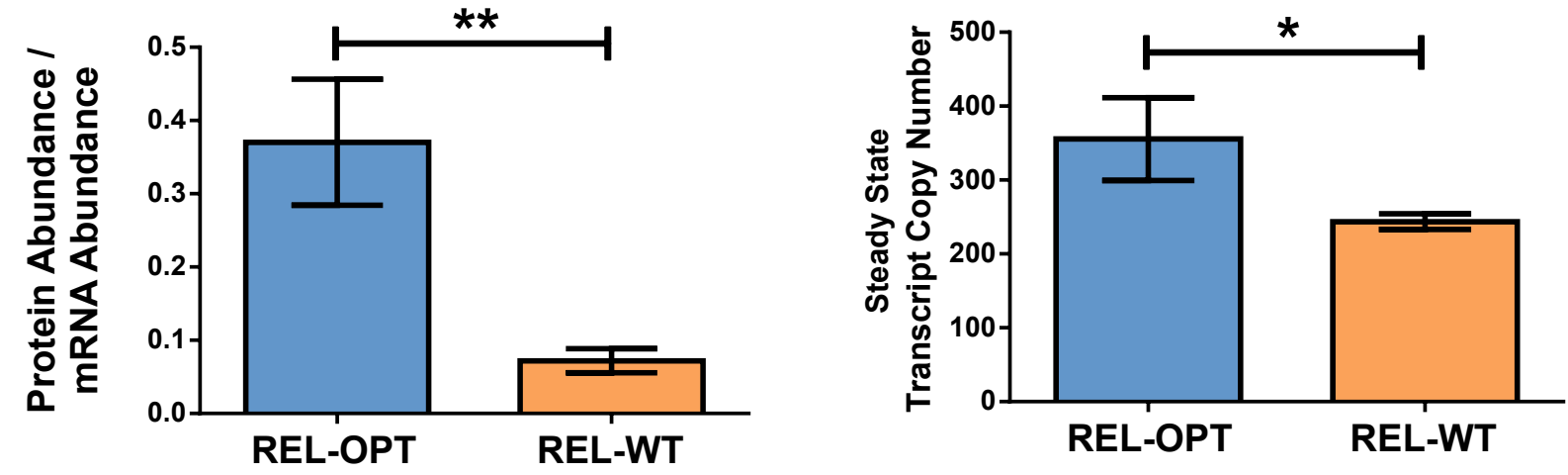

D
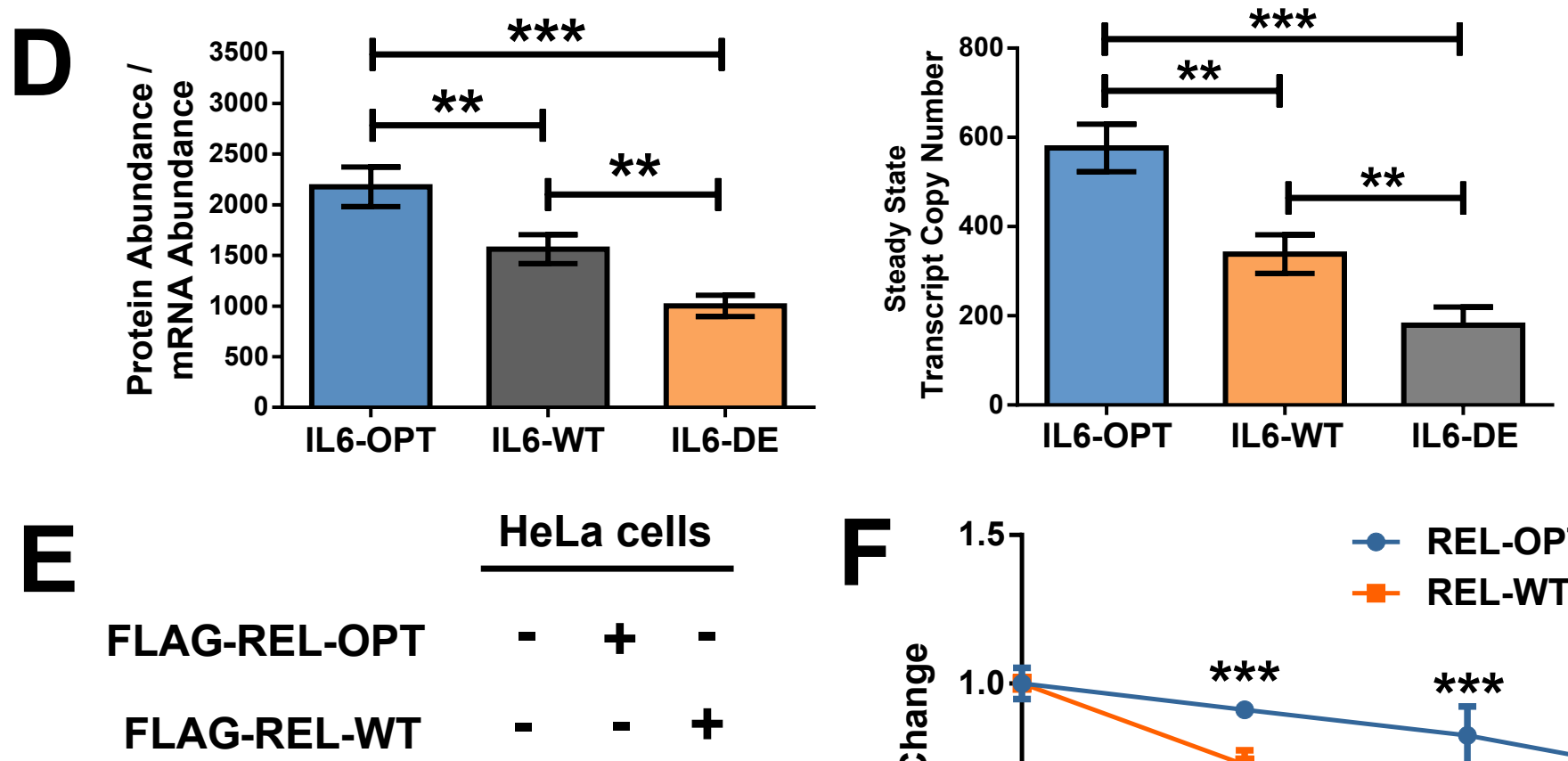

FLAG

ACTB
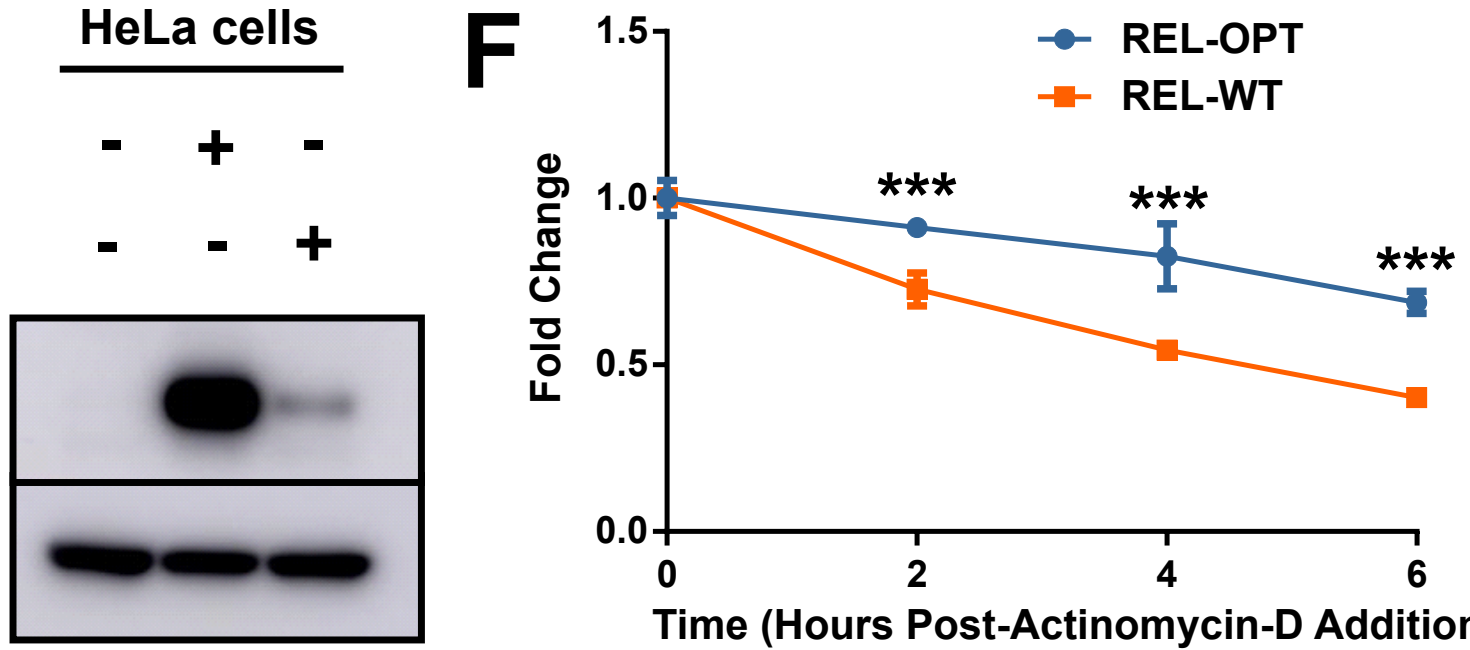

Time (Hours Post-Actinomycin-D Addition) 
A
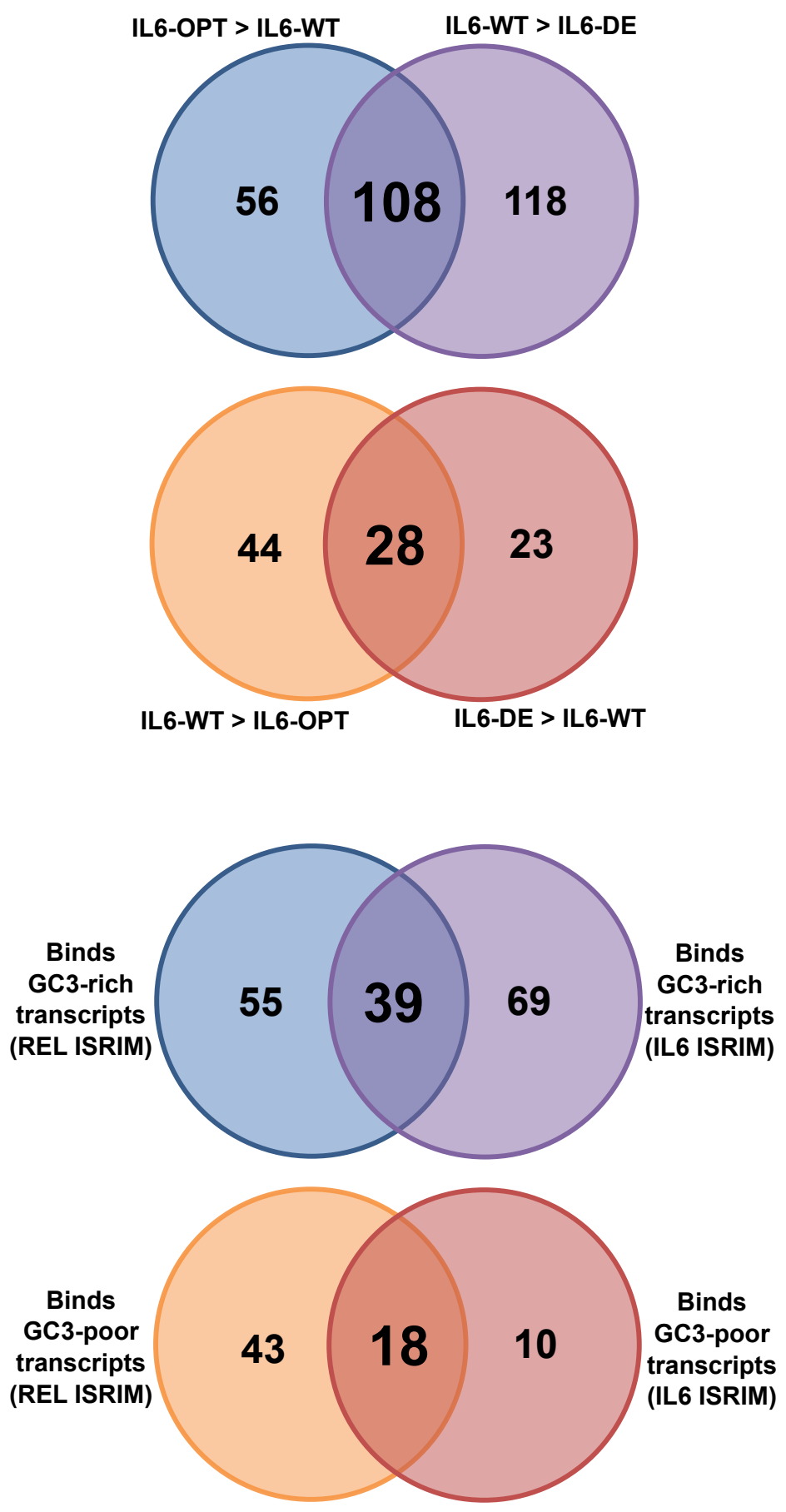


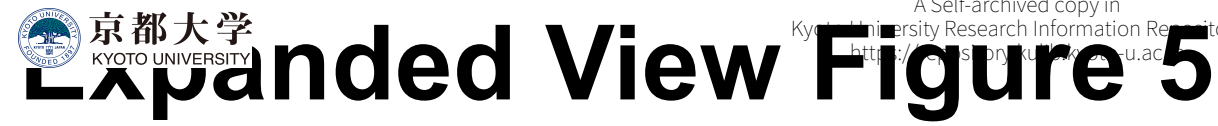

A
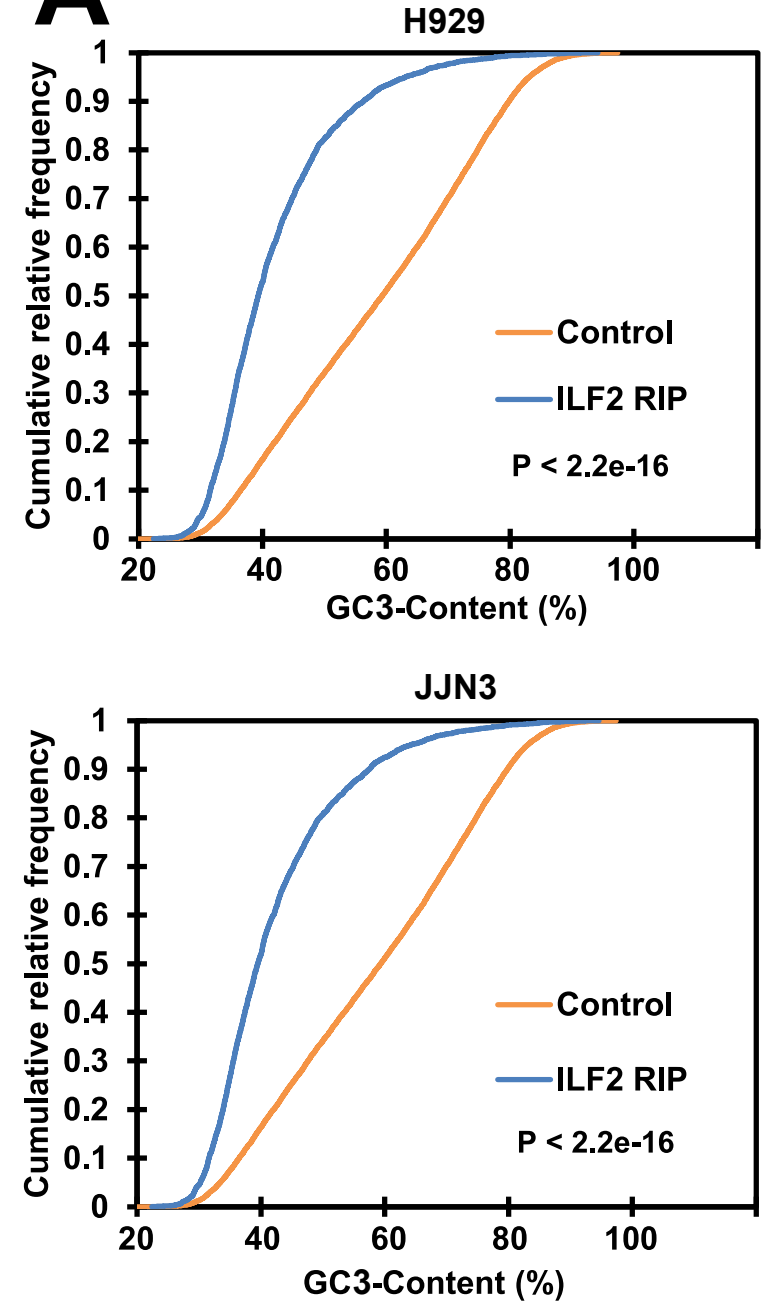

GC3-

Content (\%)
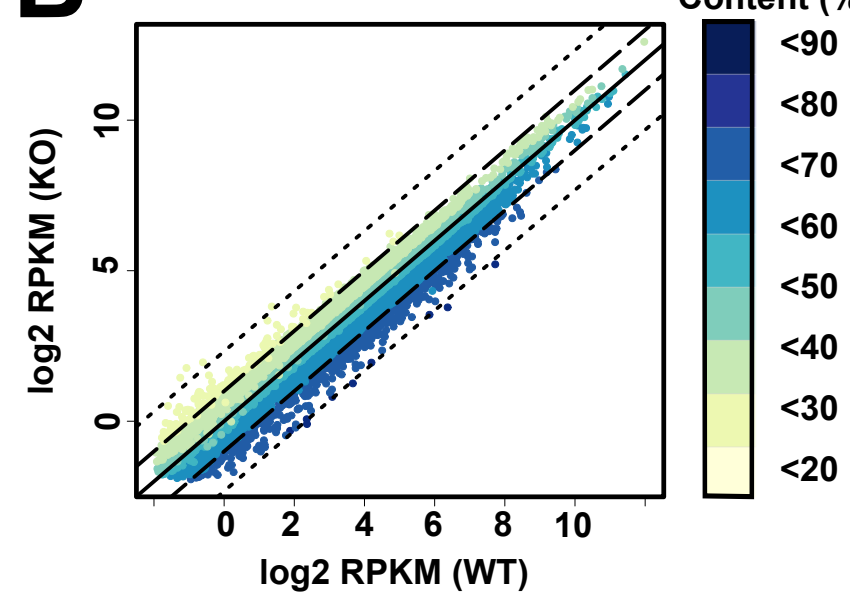

\begin{tabular}{l}
0 \\
$\frac{0}{\pi}$ \\
0 \\
$\frac{5}{0}$ \\
\hline
\end{tabular}

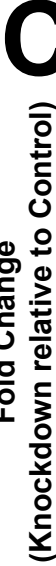

GC3-Content (\%)

100

2.0- (Knockdown relative to Control)
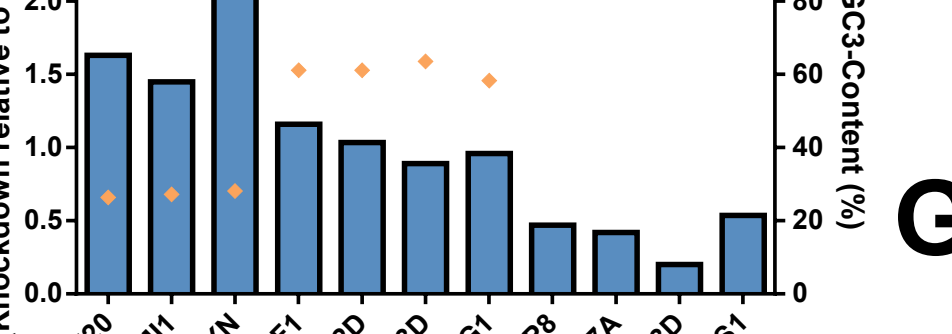

D
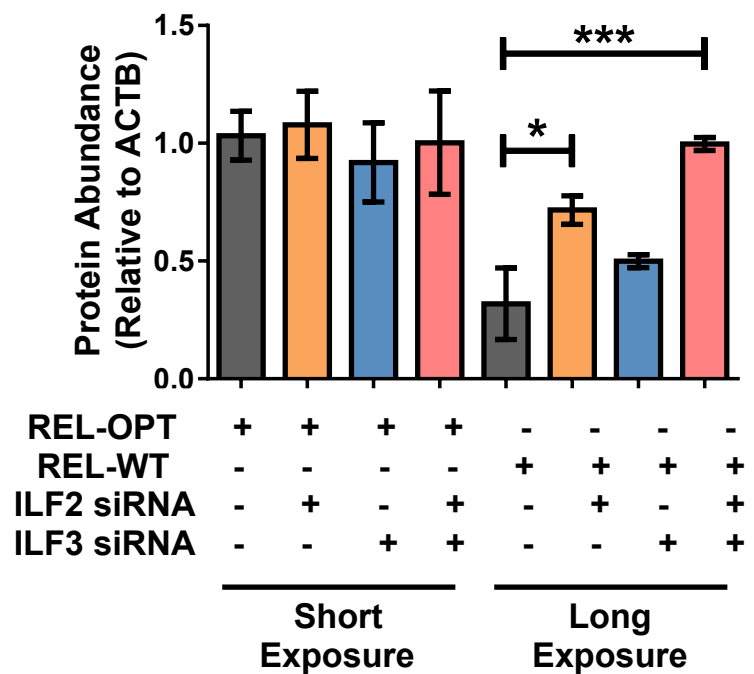

E

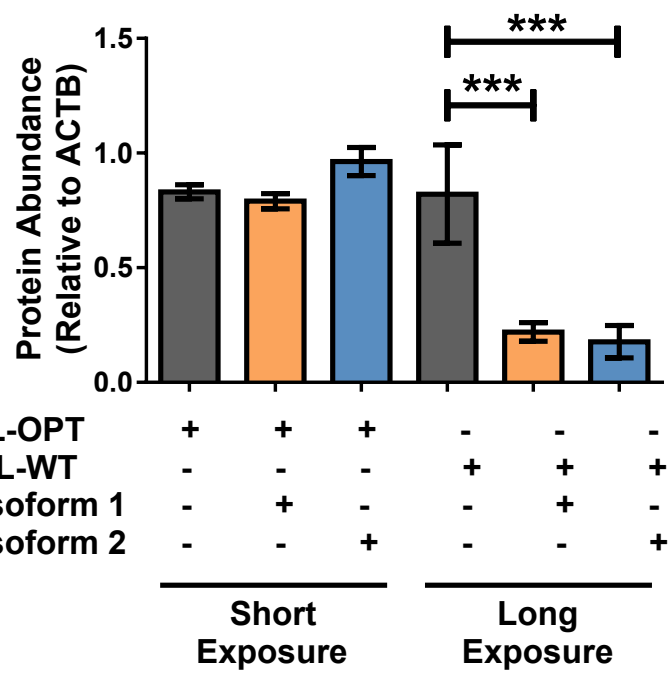

E
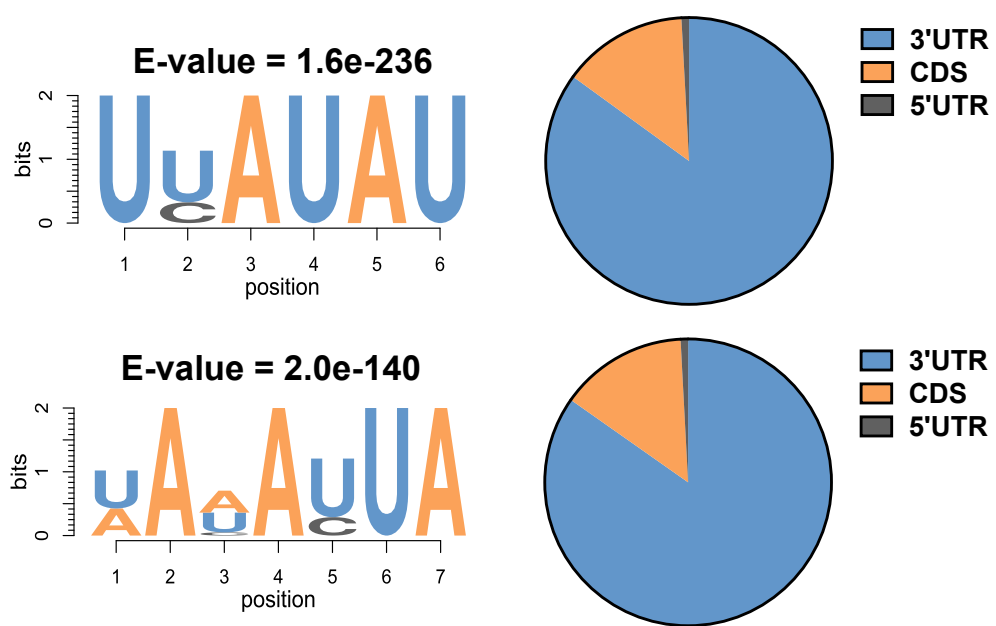

E-value $=3.5 \mathrm{e}-103$
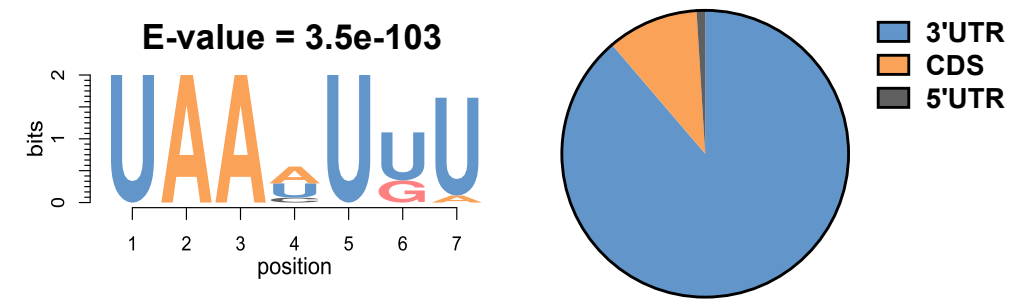

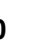

Dotu et al.
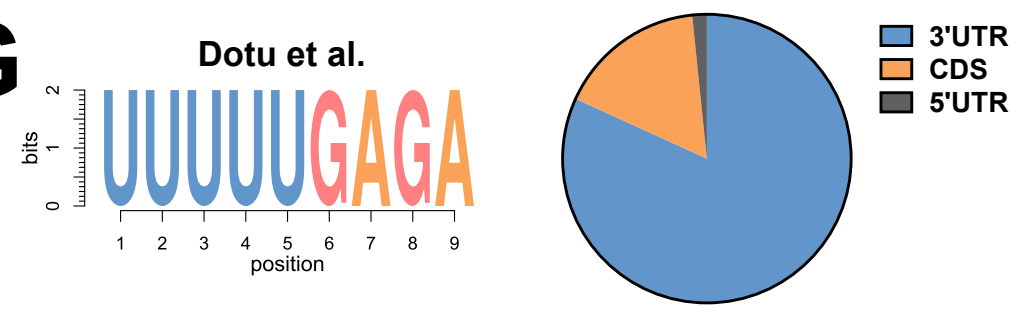